\title{
On Travelling Waves for the Stochastic Fisher-Kolmogorov-Petrovsky-Piscunov Equation
}

\author{
Joseph G. Conlon ${ }^{1}$ and Charles R. Doering ${ }^{1}$
}

Received November 26, 2004; accepted May 4, 2005

\begin{abstract}
This paper is concerned with properties of the wave speed for the stochastically perturbed Fisher-Kolmogorov-Petrovsky-Piscunov (FKPP) equation. It was shown in the classical 1937 paper by Kolmogorov, Petrovsky and Piscunov that the large time behavior of the solution to the FKPP equation with Heaviside initial data is a travelling wave. In a seminal 1995 paper Mueller and Sowers proved that this also holds for a stochastically perturbed FKPP equation. The wave speed depends on the strength $\sigma$ of the noise. In this paper bounds on the asymptotic behavior of the wave speed $c(\sigma)$ as $\sigma \rightarrow 0$ and $\sigma \rightarrow$ $\infty$ are obtained.
\end{abstract}

KEY WORDS: Stochastic pde; contact process; particle systems.

\section{INTRODUCTION}

In this paper we shall be interested in travelling wave solutions to the stochastically perturbed Kolmogorov-Petrovsky-Piscounov (FKPP) equation. The FKPP equation, ${ }^{(8,10)}$

$$
u_{t}=u_{x x}+u[1-u], \quad x \in \mathbf{R}, \quad t>0
$$

is perhaps the simplest equation which has travelling wave solutions. In fact (1.1) has a solution $u(x, t)=f_{c}(x-c t)$ for any $c \geqslant 2$, where the function $f_{c}(z)$ converges exponentially to 1 as $z \rightarrow-\infty$ and to 0 as $z \rightarrow+\infty$. In their classic 1937 paper $^{(10)}$ Kolmogorov et al. proved that if $u(x, t)$ is

\footnotetext{
${ }^{1}$ Department of Mathematics and Michigan Center for Theoretical Physics University of Michigan, Ann Arbor, MI 48109-1109; e-mail: conlon@umich.edu, doering@umich.edu
} 
the solution of (1.1) with Heaviside initial data, $u(x, 0)=1, x<0, u(x, 0)=$ $0, x>0$ then there is a function $m(t), t>0$, with the property that

$$
\begin{gathered}
\lim _{t \rightarrow \infty} m(t) / t=2, \\
\lim _{t \rightarrow \infty} \sup _{z \in \mathbf{R}}\left|u(z+m(t), t)-f_{2}(z)\right|=0 .
\end{gathered}
$$

In the 1970s and early 1980s there was much work refining the results of ref. 10 on (1.1) (see refs. 2 and 12) and also extending the results of ref. 10 to more general equations (see refs. 1 and 7). In particular the convergence (1.2) to the minimum speed wave was extended by Bramson ${ }^{(2)}$ to a convergence result for waves of any speed $c \geqslant 2$. Evidently if $c>2$ the initial data $u(z, 0)$ for $(1.1)$ must have the same asymptotic behavior when $z \rightarrow+\infty$ as the wave $f_{c}(z)$.

The fact that the wave speed for solutions of (1.1) depends on the asymptotics of the initial data is somewhat problematic if one considers (1.1) as a model for the evolution of a physical system. This could be regarded as a motivation for studying a stochastically perturbed version of (1.1). The perturbation we consider is however also a very natural extension of (1.1) from the mathematical point of view. The perturbation we shall be interested in is the equation,

$$
u_{t}=u_{x x}+u[1-u]+\sigma \sqrt{u(1-u)} W(x, t), \quad x \in \mathbf{R}, \quad t>0,
$$

where $W(x, t),(x, t) \in \mathbf{R}^{2}$, is 2-dimensional Gaussian white noise process with

$$
\langle W(x, t)\rangle=0, \quad\langle W(x, t) W(y, s)\rangle=\delta(x-y) \delta(t-s),
$$

and $\sigma \geqslant 0$ is a parameter which measures the strength of the noise. This equation has the property that the maximum principle and a duality relation which hold for (1.1) extend to (1.3).

The maximum principle for (1.3) proved by Shiga ${ }^{(18)}$ is as follows: Suppose $u_{1}(x, t)$ and $u_{2}(x, t)$ are two solutions of (1.3) with the property that $1 \geqslant u_{1}(x, 0) \geqslant u_{2}(x, 0) \geqslant 0, x \in \mathbf{R}$. Then $1 \geqslant u_{1}(x, t) \geqslant u_{2}(x, t) \geqslant 0, x \in \mathbf{R}$, for all $t>0$ with probability one. The duality relation for (1.3) was established by Shiga and Uchiyama. ${ }^{(19)}$ The definition of the dual process is quite complicated since it involves local times for Brownian motion. The duality relation for a discrete version of (1.3) (see (5.1)) is much easier to understand and is very clearly presented in ref. 5 . The duality relation consists of an identity relating expectations of polynomials in the solution $u(x, t)$ of (1.3) to expectations for a particle process. The particle 
process consists of particles diffusing on $\mathbf{R}$, with new particles being created locally at rate 1 , and intersecting particles coagulating at a rate which depends on $\sigma$. When $\sigma=0$ the particle process simply consists of branching Brownian motion, which is well known ${ }^{(2)}$ to be dual to the FKPP equation (1.1).

In 1995 Mueller and Sowers published a seminal paper ${ }^{(13)}$ establishing the existence of travelling wave solutions to (1.3). An important part of their proof is the compact support property for solutions to (1.3) with $\sigma>0$ (proved also in ref. 18). Consider a solution to (1.3) with initial data which has compact support in the sense that $u(x, 0)=1$ for $x \leqslant a(0)$, $u(x, 0)=0$ for $x \geqslant b(0)$. For $t>0$ define $a(t), b(t)$ by

$$
\begin{aligned}
& a(t)=\sup \{z \in \mathbf{R}: u(x, t)=1, x \leqslant z\}, \\
& b(t)=\inf \{z \in \mathbf{R}: u(x, t)=0, x \geqslant z\} .
\end{aligned}
$$

Then $-\infty<a(t)<b(t)<\infty$ for all $t>0$ with probability one. The main result of the Mueller-Sowers paper is as follows:

Theorem 1.1. For small $\sigma>0$ there exists a unique invariant measure for wave profiles $w(z, t)=u(z+a(t), t), z \geqslant 0$, of compact support. Further, if for the initial data one has $-\infty<a(0)<b(0)<\infty$, then there exists $c(\sigma)>0$, depending only on $\sigma$, such that

$$
\lim _{t \rightarrow \infty} \frac{b(t)}{t}=c(\sigma) \quad \text { with probability } 1 .
$$

In this paper we shall be primarily interested in the behavior of the wave speed $c(\sigma)$ as a function of $\sigma$. Our main result is the following:

Theorem 1.2. Suppose the initial data for (1.3) satisfies $-\infty<$ $a(0)<b(0)<\infty$. Then for all $\sigma>0$ there exists $c(\sigma)>0$, depending only on $\sigma$, such that

$$
\lim _{t \rightarrow \infty} \frac{b(t)}{t}=c(\sigma) \quad \text { with probability } 1 .
$$

The function $c(\sigma)$ satisfies at large $\sigma$ the inequality,

$$
\liminf _{\sigma \rightarrow \infty} \sigma^{2} c(\sigma) \geqslant 2
$$

For small $\sigma$, say $\sigma \leqslant 1 / 10$, it satisfies the inequality,

$$
2 \geqslant c(\sigma) \geqslant 2-K \log \log (1 / \sigma) /[\log \sigma]^{2},
$$

where $K>0$ is a universal constant. 
We prove the existence of the limit (1.6) by using the subadditive ergodic theorem. ${ }^{(11)}$ This avoids much of the technical difficulty in ref. 13 involved with the proof of Theorem 1.1. It does not however yield as much information. In particular a key part of the proof of Theorem 1.1 in ref. 13 is showing that $\sup [b(t)-a(t)]<\infty$ with probability one. This

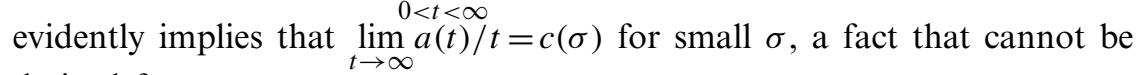
derived from our argument.

Our proof that $c(\sigma)>0$ for all $\sigma$ and the inequality (1.7) uses the duality relation for (1.3) already mentioned. We implement the duality relation by going to the discretized version (5.1) of (1.3) discussed in ref. 5, and then taking the continuum limit. Our argument therefore depends on the assumption that solutions of the discretized equation (5.1) converge in law to solutions of the continuous equation (1.3). This does not appear to have been proved in the literature. Our basic approach to proving that $c(\sigma)>0$ for all $\sigma$ and (1.7) is to use the fact that the wave speed for (1.3) is the same as the wave speed for the dual particle system. If $\sigma$ is large then the particle system is dilute. Hence the wave front for the particle system can be well approximated by a Markov chain consisting of one or two particles. An analysis of the drift of the Markov chain yields the inequality (1.7). This estimate is consistent with a conjecture (and simulations) of Doering et al. ${ }^{(5)}$ that $\lim _{\sigma \rightarrow \infty} \sigma^{2} c(\sigma)=2$.

The proof of (1.8) takes up Sections 2, 3, 4. The inequality $c(\sigma) \leqslant$ $c(0)=2$ is a simple consequence of Jensen's inequality. The lower bound on $c(\sigma)$ is more difficult to prove. Our argument is based on comparing solutions of (1.3) to a discrete version of the contact process. ${ }^{(11)} \mathrm{We}$ then adapt arguments used to prove the continuity of the wave speed for the contact process ${ }^{(11)}$ to obtain the estimate (1.8). It has previously been shown $^{(14)}$ that the scaling limit of a long range voter process yields solutions of the stochastic equation (1.3). The contact process we consider has short range interactions. The lower bound (1.8) is consistent with a conjecture of Brunet and Derrida ${ }^{(3)}$ (see also refs. 9 and 15) that there is a positive constant $K>0$ such that

$$
\lim _{\sigma \rightarrow 0}[\log \sigma]^{2}[2-c(\sigma)]=K
$$

In order to make the comparison with the contact process we need to estimate solutions of (1.3). This is done by comparing the solution of (1.3) to a solution of the equation,

$$
u_{t}=u_{x x}+u+\sigma \sqrt{u} W(x, t), \quad x \in \mathbf{R}, \quad t>0 .
$$


Equation (1.9) has the property that expectations of exponentials of linear functionals of the solution satisfy a nonlinear diffusion equation which has finite time blow up. The compact support property for solutions of (1.3) is a consequence of this finite time blow up. We shall need to estimate the size of the solution before blow up occurs, and we do this by a contraction mapping argument.

There is by now considerable literature on solutions to (1.3), (1.9) and related equations. Equation (1.9) has been particularly intensively studied because of the relation to super-Brownian motion; see, e.g. ref. 16. Other applications suggest that similar wavefront propagation questions may be of relevance for stochastic partial differential equations related to (1.3).

For one such example, it is natural to wonder about the effect of fluctuations on FKPP wavefronts if the space-time white noise in (1.3) is replaced by a colored noise of finite covariance. This is an interesting question that remains to be investigated, but we can offer a few observations. Generally such a modification will destroy the algebraic identities necessary for duality with a particle system, although if the noise is white in time but colored in space, and the equation is interpreted as the spatial continuum limit of a set of spatially discretized Itô equations, then some aspects of particle duality may still be effective if the diffusive term $u_{x x}$ is properly adapted to the noise correlations. This kind of generalization was considered by Shiga and Uchiyama, ${ }^{(19)}$ but we will not speculate on the quantitative implications of such spatial correlations for wavefronts. In another approach, if the noise is spatially white but temporally colored (and again interpreted as a limit of a spatially discrete system) then we could anticipate strong effects because for short correlation times the Stratanovich interpretation of the spatially discretized equations should be applied. These stochastic differential equations are equivalent to a set of Itô equations with additional noise-induced drift terms, and it is straightforward to see that for the nonlinear diffusion in (1.3), the noise induced drift will be affine, $\sim(1-2 u)$, with a coefficient inversely proportional to the spatial lattice spacing (at fixed $\sigma$ ). Not unexpectedly, any sensible spatial continuum limit would then very likely be either singular or trivial. For a related example that can be worked out in full detail, see ref. 4. Hence for colored noise models it is apparent that details of the application would have to be specified thoroughly and carefully in order to know how to proceed with the analysis.

Another natural question concerns the effect of fluctuations on other fundamental models for wavefronts arising in combustion or chemical kinetics. In these models the logistic drift $u(1-u)$ in FKPP is replaced with terms such as $u^{m}(1-u)$ with reaction order $m \geqslant 2$, or $e^{-E / u}(1-u)$ with activation energy $E>0$, or other qualitatively reminiscent-although 
not necessarily differentiable or even continuous-functions $f(u)$. There is a large literature on the deterministic dynamics of such systems (see, for example, refs. 6 and 21) although there are no general results for stochastic extensions. Because there is no universal way to "add noise" to these systems in order to include fluctuations in a physically meaningful way, one must revert to more primitive models where the approriate effects, due ultimately to microscopic discreteness or perhaps environmental randomness or other stochastic effects, are properly accounted for. Generally we would not expect any kind of mathematical duality as appears in the stochastic FKPP equation. Whether or not certain kinds of noise could produce similar qualitative effects as arise in the stochastic FKPP equation is presently a matter of speculation.

\section{APPROXIMATE MONOTONICITY}

We consider the solution $u(x, t), x \in \mathbf{R}, t>0$, of (1.3) with initial condition $u(x, 0), x \in \mathbf{R}$, satisfying $0 \leqslant u(x, 0) \leqslant 1, x \in \mathbf{R}$. We wish to show that in some approximate sense $u(x, t)$ increases with $t$. For an open interval $I \subset \mathbf{R}$ of length $|I|$ and a function $g: I \rightarrow \mathbf{R}$ we denote by $A v_{I}[g]$ the average of $g$ over $I$,

$$
A v_{I}[g]=\frac{1}{|I|} \int_{I} g(x) d x .
$$

Proposition 2.1. Let $u(x, t)$ be the solution of (1.3) with initial condition $u(x, 0)$. Let $I$ be the interval $I=(1,3)$. Then there exists universal constants $A, \varepsilon, C>0$ with the property: If $\eta$ satisfies $\sigma^{2}<\eta<\varepsilon<1$, and $A v_{I}[u(\cdot, 0)] \geqslant \eta$, then $A v_{I}[u(\cdot, A)]<2 \eta$ with probability smaller than $C \exp \left[-\eta / C \sigma^{2}\right]$.

We first show that the result holds when $\sigma=0$.

Lemma 2.1. If $\sigma=0$ then Proposition 2.1 holds.

Proof. Let $G(x, t)$ be the Green's function for the heat equation, where

$$
G(x, t)=\frac{1}{\sqrt{4 \pi t}} \exp \left[\frac{-x^{2}}{4 t}\right], \quad x \in \mathbf{R}, \quad t>0
$$

Then by the maximum principle one has

$$
u(x, t) \geqslant \int_{I} G(x-y, t) u(y, 0) d y .
$$


We conclude that there is a constant $c>0$ such that $u(x, 1) \geqslant c \eta, 0 \leqslant x \leqslant 4$. Let $J=[0,4]$ and $v(x, t)$ be the solution of the Dirichlet problem,

$$
\begin{aligned}
v_{t} & =v_{x x}+v(1-v), \quad x \in J, \quad t>1 \\
v(x, 1) & =c \eta, \quad x \in J, \quad v(x, t)=0, \quad x \in \partial J, \quad t>1 .
\end{aligned}
$$

Then it is clear that $u(x, t) \geqslant v(x, t), x \in J, t \geqslant 1$. We also have that $v(x, t) \leqslant c \eta e^{t-1}, x \in J, t \geqslant 1$. Suppose now $T>1$ satisfies $c \eta e^{T-1}=1 / 4$. Since $v(x, 1) \geqslant c \eta \sin (\pi x / 4)$ it follows that

$$
v(x, t) \geqslant c \eta \exp \left[\left\{\frac{3}{4}-\left(\frac{\pi}{4}\right)^{2}\right\}(t-1)\right] \sin (\pi x / 4), \quad x \in J, \quad 1 \leqslant t \leqslant T .
$$

We define the constant $A>1$ by

$$
c \exp \left[\left\{\frac{3}{4}-\left(\frac{\pi}{4}\right)^{2}\right\}(A-1)\right]=4
$$

and $\varepsilon$ by

$$
c \varepsilon e^{A-1}=1 / 4
$$

To prove Proposition 2.1 for $\sigma>0$ we shall follow the same strategy as in Lemma 2.1. First we need a stochastic estimate on solutions of (1.3). Let $J$ be an interval such that $[0,4] \subset J \subset[-1,5]$ and $u(x, t)$ be the solution of (1.3) on $J$ with Dirichlet boundary conditions on $\partial J$. Define $N(x, t)$ by

$$
N(x, t)=\int_{J} d y \int_{0}^{t} d s e^{(t-s)} G_{J}(x, y, t-s) \sqrt{u(y, s)[1-u(y, s)]} W(y, s),
$$

where $G_{J}$ is the Dirichlet Green's function on $J$. We then have the following:

Lemma 2.2. Let $T, \alpha \geqslant 1$. There is a universal constant $C>0$ such that

$$
\begin{aligned}
& P\left(\sup _{x \in J, 1 \leqslant t \leqslant T}|N(x, t)|>\alpha e^{T} \sqrt{A v_{J} u(\cdot, 0)}\right) \\
& \leqslant C \exp \left[-\frac{1}{C} \min \left\{\alpha^{2}, \alpha \sqrt{A v_{J}[u(\cdot, 0)]} / \sigma\right\}\right] .
\end{aligned}
$$


Proof. It is clear that for any $\theta \in \mathbf{R}$,

$$
\begin{aligned}
& \left\langle\operatorname { e x p } \left[\theta N(x, t)-\frac{\theta^{2}}{2} \int_{J} d y \int_{0}^{t} d s e^{2(t-s)} G_{J}^{2}(x, y, t-s)\right.\right. \\
& \quad \times u(y, s)[1-u(y, s)]]\rangle=1 .
\end{aligned}
$$

Let $V(x, t)$ be defined by

$$
V(x, t)=\int_{J} d y \int_{0}^{t} d s e^{2(t-s)} G_{J}^{2}(x, y, t-s) u(y, s) .
$$

Then it follows from (2.4) that for any $\theta \in \mathbf{R}$,

$$
\langle\exp [\theta N(x, t)]\rangle \leqslant\left\langle\exp \left[2 \theta^{2} V(x, t)\right]\right\rangle
$$

Now the expectation on the RHS of (2.5) is to be computed for $u(x, t)$ the solution to (1.3) on $J$ with Dirichlet boundary conditions and initial data $u(x, 0), x \in J$. Observe that this expectation only increases if we assume $u(x, t)$ is the solution to the equation,

$$
u_{t}=u_{x x}+u+\sigma \sqrt{u} W
$$

on $J$ with Dirichlet boundary conditions and initial data $u(x, 0), x \in J$.

Let $\varphi: J \times(0, \infty) \rightarrow \mathbf{R}$ be nonnegative and consider the variable $V_{\varphi}(t)$,

$$
V_{\varphi}(t)=\int_{J} d y \int_{0}^{t} d s \varphi(y, s) u(y, s)
$$

where $u(x, t)$ is the solution to (2.6) on $J$ with Dirichlet boundary conditions and initial data $u(\cdot, 0)$. It is well known that

$$
\left\langle\exp \left[V_{\varphi}(t)\right]\right\rangle=\exp \left[\int_{J} u(x, 0) v(x, 0) d x\right]
$$

where $v(x, s), s \leqslant t, x \in J$, satisfies the initial-boundary value problem,

$$
\begin{gathered}
\frac{\partial v}{\partial s}+v_{x x}+v+\frac{\sigma^{2}}{2} v^{2}+\varphi=0, \quad s<t, \\
v(x, t)=0, \quad x \in J, \quad v(x, s)=0, \quad x \in \partial J, \quad s<t .
\end{gathered}
$$


Let $X(s), s \geqslant 0$, denote Brownian motion with diffusion constant 1 . For a function $v(x, s), 0 \leqslant s \leqslant t, x \in J$, define $T v(x, s), 0 \leqslant s \leqslant t, x \in J$, by

$$
\begin{aligned}
& T v(x, s) \\
= & \int_{s}^{t} d s^{\prime} e^{s^{\prime}-s} E\left[\exp \left\{\frac{\sigma^{2}}{2} \int_{s}^{s^{\prime}} v\left(X\left(s^{\prime \prime}\right), s^{\prime \prime}\right) d s^{\prime \prime}\right\} \varphi\left(X\left(s^{\prime}\right), s^{\prime}\right) ; \tau>s^{\prime} \mid X(s)=x\right],
\end{aligned}
$$

where $\tau$ is the first exit time from $J$. (Here we use the standard notation $E[\cdot]$ for the expectation over Brownian motion.) Then the solution to (2.8) satisfies $v=T v$. Suppose now that for some $x^{\prime} \in \mathbf{R}$, the function $\varphi$ satisfies the inequality,

$$
|\varphi(y, s)| \leqslant \frac{e^{2(t-s)}}{\beta \sqrt{t-s}} G\left(x^{\prime}-y, t-s\right), \quad y \in J, \quad 0 \leqslant s \leqslant t,
$$

where $\beta>0$ is a constant. We shall show that if $\beta$ is sufficiently large then $T$ is a contraction mapping. In fact for a function $v: J \times[0, t] \rightarrow \mathbf{R}$ let

$$
\|v\|=\sup _{x \in J, 0 \leqslant s \leqslant t} e^{-2(t-s)}|v(x, s)| .
$$

Then from (2.10) it follows that

$$
\|T v\| \leqslant \frac{1}{\beta \sqrt{\pi}} \exp \left[\frac{\sigma^{2}}{4} e^{2 t}\|v\|\right] .
$$

Let $\mathcal{S}_{\beta}=\{v: J \times[0, t] \rightarrow \mathbf{R}:\|v\| \leqslant 1 / \beta\}$. Then if $\beta \geqslant \sigma^{2} e^{2 t} / 2$ it follows from (2.11) that $T$ maps $\mathcal{S}_{\beta}$ into $\mathcal{S}_{\beta}$. One can see furthermore that $T$ is also a contraction mapping whence there is a unique solution $v \in \mathcal{S}_{\beta}$ to the equation $v=T v$. We conclude therefore that if $\varphi$ satisfies (2.10) then

$$
\left\langle\exp \left[V_{\varphi}(t)\right]\right\rangle \leqslant \exp \left[4 e^{2 t} A v_{J}[u(\cdot, 0)] / \beta\right],
$$

provided $\beta \geqslant \sigma^{2} e^{2 t} / 2$. Now we have that

$$
\begin{aligned}
& P\left(|N(x, t)|>\alpha e^{t} \sqrt{A v_{J}[u(\cdot, 0)]}\right) \\
& \quad \leqslant \exp \left[-\theta \alpha e^{t} \sqrt{A v_{J}[u(\cdot, 0)]}\right]\{\langle\exp [\theta N(x, t)]\rangle+\langle\exp [-\theta N(x, t)]\rangle\}
\end{aligned}
$$

for any $\theta>0$. 
From (2.5), (2.12) it therefore follows that

$$
\begin{aligned}
& P\left(|N(x, t)|>\alpha e^{t} \sqrt{A v_{J}[u(\cdot, 0)]}\right) \\
& \quad \leqslant 2 \exp \left[-\theta \alpha e^{t} \sqrt{A v_{J} u(\cdot, 0)}+4 \theta^{2} e^{2 t} A v_{J}[u(\cdot, 0)] / \sqrt{\pi}\right],
\end{aligned}
$$

provided $\theta^{2} e^{2 t} \leqslant 2 \sqrt{\pi} / \sigma^{2}$. The minimum of the RHS of (2.13) occurs at $\theta=$ $\alpha \sqrt{\pi} / 8 e^{t} \sqrt{A v_{J}[u(\cdot, 0)]}$, in which case the RHS of $(2.13)$ is $2 \exp \left[-\alpha^{2} \sqrt{\pi} / 16\right]$. On the other hand if we put $\theta^{2} e^{2 t}=2 \sqrt{\pi} / \sigma^{2}$, the RHS of (2.13) is given by

$$
2 \exp \left[-\alpha 2^{1 / 2} \pi^{1 / 4} \sqrt{A v_{J}[u(\cdot, 0)]} / \sigma+8 A v_{J}[u(\cdot, 0)] / \sigma^{2}\right]
$$

We conclude then that

$$
\begin{aligned}
& P\left(|N(x, t)|>\alpha e^{t} \sqrt{A v_{J}[u(\cdot, 0)]}\right) \\
& \quad \leqslant 2 \exp \left[-\frac{1}{16} \min \left\{\alpha^{2}, \alpha \sqrt{A v_{J}[u(\cdot, 0)]} / \sigma\right\}\right] .
\end{aligned}
$$

Next we obtain an estimate on the difference $N(x, t)-N(x, t-\delta)$ where $t \geqslant 1,0<\delta<1 / 10$. Similarly to the derivation of $(2.5)$ we have that

$$
\langle\exp [\theta\{N(x, t)-N(x, t-\delta)\}]\rangle \leqslant\left\langle\exp \left[V_{\varphi}(t)\right]\right\rangle,
$$

where $\varphi(y, s)$ is given by

$$
\begin{gathered}
\varphi(y, s)=2 \theta^{2} e^{2(t-s)} G_{J}^{2}(x, y, t-s), \quad y \in J, \quad t-\delta<s<t, \\
\varphi(y, s)=2 \theta^{2}\left\{e^{(t-s)} G_{J}(x, y, t-s)-e^{t-\delta-s} G_{J}(x, y, t-\delta-s)\right\}^{2}, \\
y \in J, 0<s<t-\delta .
\end{gathered}
$$

It follows that

$$
\begin{gathered}
\varphi(y, s) \leqslant \frac{2 \theta^{2}}{\sqrt{\pi(t-s)}} G(x-y, t-s), \quad y \in J, \quad t-\delta<s<t, \\
\varphi(y, s) \leqslant 4 \theta^{2}\left\{\frac{1}{\sqrt{\pi(t-s)}} G(x-y, t-s)+\frac{1}{\sqrt{\pi(t-\delta-s)}} G(x-y, t-\delta-s)\right\}, \\
y \in J, \quad t-2 \delta<s<t-\delta,
\end{gathered}
$$




$$
\begin{gathered}
\varphi(y, s) \leqslant C \theta^{2} e^{2(t-s)} \delta^{2}\left\{(t-s)^{-5 / 2}+(t-s)^{-1 / 2}\right\} G(x-y, 2(t-s)), \\
0<s<t-2 \delta
\end{gathered}
$$

where $C$ is a universal constant. For a function $v: J \times[0, t] \rightarrow \mathbf{R}$ define $\|v\|_{\delta}$ by

$$
\|v\|_{\delta}=\sup _{x \in J, 0 \leqslant s \leqslant t}(t+\delta-s)^{1 / 2} e^{-2(t-s)}|v(x, s)| .
$$

Then taking $\varphi$ to be the function (2.16) it follows from the inequalities (2.17) that if $T$ is the operator (2.9) then

$$
\|T v\|_{\delta} \leqslant C_{1} \theta^{2} \delta^{1 / 2} \exp \left[C_{1} \sigma^{2}\|v\|_{\delta} e^{2 t}\right]
$$

for some universal constant $C_{1}$. Let $\mathcal{S}_{\theta, \delta}$ be the set,

$$
\mathcal{S}_{\theta, \delta}=\left\{v: J \times[0, t] \rightarrow \mathbf{R}:\|v\|_{\delta} \leqslant 2 C_{1} \theta^{2} \delta^{1 / 2}\right\}
$$

It is clear that if $C_{1}^{2} \theta^{2} \sigma^{2} e^{2 t} \delta^{1 / 2} \leqslant 1 / 4$, then $T$ maps $\mathcal{S}_{\theta, \delta}$ to $\mathcal{S}_{\theta, \delta}$. One can also see that $T$ is a contraction mapping, whence there is a unique solution $v \in \mathcal{S}_{\theta, \delta}$ to the equation $v=T v$. It follows then from (2.7), (2.15) that

$$
\langle\exp [\theta\{N(x, t)-N(x, t-\delta)\}]\rangle \leqslant \exp \left[8 C_{1} \theta^{2} \delta^{1 / 2} e^{2 t} A v_{J}[u(\cdot, 0)]\right],
$$

provided $C_{1}^{2} \theta^{2} \delta^{2} e^{2 t} \delta^{1 / 2} \leqslant 1 / 4$. Arguing as before we conclude that

$$
\begin{aligned}
& P\left(|N(x, t)-N(x, t-\delta)|>\alpha e^{t} \sqrt{A v_{J}[u(\cdot, 0)]}\right) \\
& \quad \leqslant 2 \exp \left[\frac{-1}{32 C_{1}} \min \left\{\frac{\alpha^{2}}{\delta^{1 / 2}}, \alpha \sqrt{A v_{J}[u(\cdot, 0)]} / \delta^{1 / 4} \sigma\right\}\right] .
\end{aligned}
$$

We can make a similar argument to estimate the difference $N\left(x+\delta^{1 / 2}, t\right)-$ $N(x, t)$. Thus we have

$$
\begin{aligned}
& P\left(\left|N\left(x+\delta^{1 / 2}, t\right)-N(x, t)\right|>\alpha e^{t} \sqrt{A v_{J}[u(\cdot, 0)]}\right) \\
& \quad \leqslant 2 \exp \left[\frac{-1}{32 C_{1}} \min \left\{\frac{\alpha^{2}}{\delta^{1 / 2}}, \alpha \sqrt{A v_{J} u(\cdot, 0)} / \delta^{1 / 4} \sigma\right\}\right] .
\end{aligned}
$$


The result follows now from (2.14), (2.18), (2.19) by the usual dyadic decomposition method. ${ }^{(20)}$ That is one first decomposes the rectangle $J \times$ $[1, T]$ dyadically. Suppose one has an estimate on the probability that the maximum of $N(x, t)$ on all corners of dyadic rectangles of a given generation is large. Then one makes use of the inequalities (2.18), (2.19) to obtain an estimate on the probability that the maximum of $N(x, t)$ on all corners of dyadic rectangles of the next generation is slightly larger.

Proof of Proposition 2.1. Let $v(x, t)$ be the solution to the stochastic Dirichlet problem,

$$
\begin{aligned}
v_{t} & =v_{x x}+v(1-v)+\sqrt{v(1-v)} W, \quad x \in J, \quad t>0, \\
v(x, 0) & =u(x, 0), \quad x \in I, \quad v(x, 0)=0, \quad x \in J \backslash I, \\
v(x, t) & =0, \quad x \in \partial J, \quad t>0
\end{aligned}
$$

where $J$ is the interval $J=[-1,5]$. Then $u(x, t) \geqslant v(x, t), x \in J, t>0$, and $v(x, t)$ satisfies the stochastic integral equation,

$$
\begin{aligned}
v(x, t)= & e^{\gamma t} \int_{I} G_{J}(x, y, t) u(y, 0) d y \\
& +\int_{J} d y \int_{0}^{t} d s e^{\gamma(t-s)} G_{J}(x, y, t-s) v(y, s)[1-\gamma-v(y, s)] \\
& +\sigma N_{\gamma}(x, t)
\end{aligned}
$$

where

$$
N_{\gamma}(x, t)=\int_{J} d y \int_{0}^{t} d s e^{\gamma(t-s)} G_{J}(x, y, t-s) \sqrt{v(y, s)[1-v(y, s)]} W(y, s)
$$

Taking $\gamma=1$ in (2.20) and using Lemma 2.2 we see that for $T>1$ there are universal constants $C_{1}, C_{2}>0$ such that $\sup _{x \in J, 1 \leqslant t \leqslant T} v(x, t) \leqslant C_{1} \eta e^{T}$ with probability at least $1-C_{2} \exp \left[-\eta / \sigma^{2} C_{2}\right]$. If we take $\gamma=0$ in (2.20) and again use Lemma 2.2 we see that $v(x, 1) \geqslant c \eta, x \in[0,4]$ for some universal $c>0$ with probability at least $1-C_{2} \exp \left[-\eta / C_{2} \sigma^{2}\right]$. Next we consider the stochastic Dirichlet problem on the interval $J_{1}=[0,4]$,

$$
\begin{aligned}
w_{t} & =w_{x x}+w(1-w)+\sqrt{w(1-w)} W, \quad x \in J_{1}, \quad t>1, \\
w(x, 1) & =c \eta, \quad x \in J_{1}, \quad w(x, t)=0, \quad x \in \partial J_{1}, \quad t>1 .
\end{aligned}
$$


It follows from our estimates on $v$ that with probability at least $1-$ $2 C_{2} \exp \left[-\eta / C_{2} \sigma^{2}\right]$ one has

$$
w(x, t) \leqslant v(x, t), \quad x \in J_{1}, \quad t \geqslant 1, \sup _{x \in J_{1}, 1 \leqslant t \leqslant T} w(x, t) \leqslant C_{1} \eta e^{T} .
$$

Now $w(x, t)$ satisfies the stochastic integral equation,

$$
\begin{aligned}
w(x, t)= & e^{3(t-1) / 4} \int_{J_{1}} G_{J_{1}}(x, y, t-1) c \eta d y \\
& +\int_{J_{1}} d y \int_{1}^{t} d s e^{3(t-s) / 4} G_{J_{1}}(x, y, t-s) w(y, s)[1 / 4-w(y, s)] \\
& +\sigma N(x, t)
\end{aligned}
$$

where

$$
\begin{aligned}
N(x, t)= & \int_{J_{1}} d y \int_{1}^{t} d s e^{3(t-s) / 4} G_{J_{1}}(x, y, t-s) \\
& \times \sqrt{w(y, s)[1-w(y, s)]} W(y, s) .
\end{aligned}
$$

Define now $A \geqslant 2$ by (2.2) and $\varepsilon$ similarly to (2.3) by

$$
C_{1} \varepsilon e^{A}=1 / 4 \text {. }
$$

By Lemma 2.2 one has that

$$
P\left(\sup _{x \in J_{1}}|N(x, A)|>\eta / \sigma\right) \leqslant C_{3} \exp \left[-\eta / C_{3} \sigma^{2}\right] .
$$

The result follows from this last inequality, (2.21), (2.22).

\section{LOWER BOUND ON THE WAVE SPEED}

In this section we obtain a lower bound on the wave speed provided $\sigma$ is sufficiently small. To do this we shall compare the solutions $u(x, t)$ of (1.3) to a discrete time version of the contact process. For any $A \geqslant 1, \eta$ such that $0<\eta<1$ and solution $u(x, t)$ of (1.3) we define random variables $\xi_{m}(n), n, m \in \mathbf{Z}, m \geqslant 0$, as follows:

$$
\begin{array}{ll}
\xi_{m}(n)=1 & \text { if } A v_{(2 n, 2 n+2)}[u(\cdot, m A)] \geqslant \eta, \\
\xi_{m}(n)=0 & \text { if } A v_{(2 n, 2 n+2)}[u(\cdot, m A)]<\eta .
\end{array}
$$


Next let $q$ satisfy $0<q<1$. We define a Markov chain $\zeta_{m}: \mathbf{Z} \rightarrow\{0,1\}, m=$ $0,1,2, \ldots$ with transition probabilities determined by $q$. Let $A=\{n \in \mathbf{Z}$ : $\left.\zeta_{m}(n)=1\right\}$ and $X_{n}, n \in A$, be i.i.d. Bernoulli variables with $P\left(X_{n}=0\right)=q$. Then $\zeta_{m+1}$ is defined as follows:

$$
\begin{array}{cc} 
& \zeta_{m+1}(n)=1 \quad \text { if } n \in A \text { and } X_{n}=1 \\
n-1 \in A \quad \text { and } \quad X_{n-1}=1 \text { or } n+1 \in A \text { and } X_{n+1}=1 ; \\
\zeta_{m+1}(n)=0, \quad \text { otherwise. }
\end{array}
$$

Our first lemma is a version of the duality theorem for the contact process, Theorem 1.7 of chapter VI of ref. 11.

Lemma 3.1. Assume $A, \varepsilon, \eta$ are as in Proposition 2.1. Let $C \geqslant 1$ and $\zeta_{m}$ be the Markov chain of (3.2) with $q=\exp \left[-\eta / C \sigma^{2}\right]$. Let $0<\delta<1$ and $\xi_{m}$ be the variables (3.1) corresponding to a solution of (1.3) with $\sigma^{2}<\delta \eta$. The constants $C, \delta$ can be chosen universally so that for $m \geqslant 0$,

$$
P\left(\sum_{n \in \mathbf{Z}} \xi_{m}(n) \zeta_{0}(n)=0\right) \leqslant P\left(\sum_{n \in \mathbf{Z}} \xi_{0}(n) \zeta_{m}(n)=0\right)
$$

Proof. We first prove (3.3) for $m=1$. Let $S_{1}$ be the set,

$$
S_{1}=\left\{n \in \mathbf{Z}: \zeta_{0}(n)=1, \quad \xi_{0}(n-1)+\xi_{0}(n)+\xi_{0}(n+1)>0\right\}
$$

If $\left|S_{1}\right|$ denotes the number of elements of $S_{1}$, then

$$
P\left(\sum_{n \in \mathbf{Z}} \xi_{0}(n) \zeta_{1}(n)=0\right)=q^{\left|S_{1}\right|}
$$

Next let $S_{2}$ be the set,

$$
S_{2}=\left\{n \in \mathbf{Z}: \xi_{0}(n)=1, \quad \zeta_{0}(n-1)+\zeta_{0}(n)+\zeta_{0}(n+1)>0\right\}
$$

Evidently one has that $2\left|S_{2}\right| \geqslant\left|S_{1}\right|$. Also there exists a subset $S_{3} \subset S_{2}$ such that $3\left|S_{3}\right| \geqslant\left|S_{2}\right|$ and the intervals $(2 n-2,2 n+4), n \in S_{3}$, are non-overlapping. Now from Proposition 2.1 there is the inequality,

$$
P\left(\sum_{n \in \mathbf{Z}} \xi_{1}(n) \zeta_{0}(n)=0\right) \leqslant\left\{C \exp \left[-\eta / C \sigma^{2}\right]\right\}^{\left|S_{3}\right|}
$$

The inequality (3.3) for $m=1$ follows from (3.4), (3.5) by choosing $\delta$ sufficiently small. The argument extends to all $m \geqslant 1$ by induction. 
Next, as in Chapter VI, Section 1 of $^{(11)}$ we construct a probability measure $v$ on the configurations of $\xi_{0}$ which has the property that,

$$
\begin{aligned}
& \int P\left(\sum_{n \in \mathbf{Z}} \xi_{0}(n) \zeta_{m}(n)=0\right) d \nu\left(\xi_{0}\right) \\
& \leqslant \int P\left(\sum_{n \in \mathbf{Z}} \xi_{0}(n) \zeta_{0}(n)=0\right) d \nu\left(\xi_{0}\right), \quad m \geqslant 1
\end{aligned}
$$

for any initial configuration $\zeta_{0}$ of the Markov chain $\zeta_{m}, m \geqslant 0$. Let $f(k), k=1,2, \ldots$, be a probability density with finite mean, whence

$$
\sum_{k=1}^{\infty} f(k)=1, \quad \sum_{k=1}^{\infty} k f(k)<\infty .
$$

Then $v$ is defined in terms of $f$ as follows: Suppose $a_{i} \in \mathbf{Z}, 1 \leqslant i \leqslant N$ with $a_{1}<a_{2}<\cdots<a_{N}$. There is the formula,

$$
\begin{aligned}
v & \left\{\xi_{0}: \xi_{0}\left(a_{i}\right)=1,1 \leqslant i \leqslant N, \xi_{0}(n)=0, a_{1}<n<a_{N}, n \neq a_{j}, 2 \leqslant j \leqslant N-1\right\} \\
& =\prod_{i=1}^{N-1} f\left(a_{i+1}-a_{i}\right) / \sum_{k=1}^{\infty} k f(k) .
\end{aligned}
$$

Let $h(z), z \in \mathbf{C},|z|<1$, be the function,

$$
h(z)=\sum_{k=1}^{\infty} \frac{z^{k-1}}{k^{2}} .
$$

We consider $f(k), k=1,2, \ldots$, of the form

$$
f(k)=(\Lambda q)^{k-1} / k^{2} h(\Lambda q), k=1,2, \ldots
$$

where $0<\Lambda q$ and $q$ is the parameter determining the Markov chain $\zeta_{m}$ of (3.2).

Lemma 3.2. There exist universal constants $\Lambda \geqslant 1$ and $\varepsilon, 0<\varepsilon<1$, $\Lambda \varepsilon \leqslant 1 / 2$, such that if $0<q \leqslant \varepsilon$ then the measure (3.7) determined by (3.8) satisfies the inequality (3.6) for arbitrary initial configurations $\zeta_{0}$. 
Proof. It is sufficient to prove (3.6) for $m=1$, so we shall assume wlog that $m=1$. Suppose first that for some $N \geqslant 1$ the set $\left\{n \in \mathbf{Z}: \zeta_{0}(n)=\right.$ $1\}=\{1,2, \ldots, N\}$. Then the RHS of (3.6) is given by

$$
v\left\{\xi_{0}: \xi_{0}(n)=0, \quad 1 \leqslant n \leqslant N\right\}=\sum_{k=1}^{\infty} k f(N+k) / \sum_{k=1}^{\infty} k f(k) .
$$

From (3.8) and the fact that $\Lambda q \leqslant 1 / 2$ we conclude

$$
\nu\left\{\xi_{0}: \xi_{0}(n)=0, \quad 1 \leqslant n \leqslant N\right\} \geqslant c(\Lambda q)^{N} / N^{2}
$$

for some universal constant $c>0$. The LHS of (3.6) is bounded above by

$$
\begin{aligned}
& v\left\{\xi_{0}: \xi_{0}(n)=0,0 \leqslant n \leqslant N+1\right\} \\
& +\sum_{j=0}^{N+1} v\left\{\xi_{0}: \xi_{0}(j)=1, \xi_{0}(n)=0,0 \leqslant n \leqslant N+1, n \neq j\right\} P\left(\zeta_{1}(j)=0\right) \\
& +\sum_{\substack{0 \leqslant j_{1}<j_{2} \leqslant N+1 \\
\quad}} v\left\{\xi_{0}: \xi_{0}\left(j_{1}\right)=\xi_{0}\left(j_{2}\right)=1, \xi_{0}(n)=0,0 \leqslant n \leqslant N+1, n \neq j_{1}, j_{2}\right\} \\
& \quad \times\left(\zeta_{1}\left(j_{1}\right)=\zeta_{1}\left(j_{2}\right)=0\right)+\cdots
\end{aligned}
$$

It is clear that there is a universal constant $C>0$ such that the first term in (3.10) is bounded as

$$
\nu\left\{\xi_{0}: \xi_{0}(n)=0,0 \leqslant n \leqslant N+1\right\} \leqslant C(\Lambda q)^{N+2} / N^{2} .
$$

Let $C_{1}$ be a constant which satisfies

$$
\sum_{k=1}^{r-1} \frac{1}{k^{2}} \frac{1}{(r-k)^{2}} \leqslant \frac{C_{1}}{r^{2}}, \quad r=2,3, \ldots
$$

Then one can see that there is a universal constant $C>0$ such that the $(m+1)$ st term in (3.10) satisfies the inequality,

$$
\sum_{0 \leqslant j_{1}<j_{2}<\cdots<j_{m} \leqslant N+1} \leqslant C\left(\frac{C_{1}}{\Lambda}\right)^{m} \frac{(\Lambda q)^{N+2}}{N^{2}},
$$

provided $m \leqslant N$. For $m=N+1, N+2$ there are the inequalities, 


$$
\begin{gathered}
\sum_{0 \leqslant j_{1}<j_{2}<\cdots<j_{N+1} \leqslant N+1} \leqslant C\left(\frac{C_{1}}{\Lambda}\right)^{N} \frac{(\Lambda q)^{N+1}}{N^{2}}, \\
\sum_{0 \leqslant j_{1}<j_{2}<\cdots<j_{N+2} \leqslant N+1} \leqslant C\left(\frac{C_{1}}{\Lambda}\right)^{N} \frac{(\Lambda q)^{N}}{N^{2}} .
\end{gathered}
$$

If we choose $\Lambda=2 C_{1}$ and $\varepsilon$ sufficiently small the sum of all the terms in (3.10) corresponding to the LHS of (3.11) with $m \leqslant N$ is bounded above by $1 / 2$ the RHS of (3.9). Similarly the term of (3.10) corresponding to the LHS of (3.12) is bounded above by $1 / 4$ the RHS of (3.9). Finally the term of (3.10) corresponding to the LHS of (3.13) is bounded above by $1 / 4$ the RHS of (3.9) if we choose $\Lambda \geqslant 2 C_{1}$ sufficiently large in a universal way. We have proved (3.6) in the case when $A=\left\{n \in \mathbf{Z}: \zeta_{0}(n)=1\right\}$ is given by $A=\{1,2, \ldots, N\}$.

Next we generalize the previous argument to any finite set $A$. Let $\tilde{A}$ be the set of neighbors of $A, \tilde{A}=\{n \in \mathbf{Z}: n \in A$ or $n-1 \in A$ or $n+1 \in A\}$. We assume $\tilde{A}$ is the union of $k$ intervals $\left[\ell_{j}, r_{j}\right] \cap \mathbf{Z}, 1 \leqslant j \leqslant k$, where $r_{j}+$ $1<\ell_{j+1}, j=1, \ldots, k-1$. Note that since $\tilde{A}$ is formed from $A$ the length of each interval is at least 2 . We show that there is a universal constant $C$ such that

$$
v\left\{\xi_{0}: \xi_{0}(n)=0, n \in \tilde{A}\right\} \leqslant C^{k}(\Lambda q)^{2 k} v\left\{\xi_{0}: \xi_{0}(n)=0, n \in A\right\}
$$

We prove (3.14) by showing that for any $\xi_{0}$ satisfying $\xi_{0}(n)=0, n \in \tilde{A}$ there is a configuration $T \xi_{0}$ satisfying $T \xi_{0}(n)=0, n \in A$ and

$$
\nu\left\{\xi_{0}\right\} \leqslant C^{k}(\Lambda q)^{2 k} \nu\left\{T \xi_{0}\right\}
$$

To define $T \xi_{0}$ we observe that there are integers $a_{1}, \ldots, a_{k}, b_{1}, \ldots, b_{k}$ satisfying

$$
b_{1} \leqslant \ell_{1}<r_{1} \leqslant a_{1}<b_{2} \leqslant \ell_{2}<r_{2} \leqslant a_{2}<\cdots<b_{k} \leqslant \ell_{k}<r_{k} \leqslant a_{k},
$$

such that $\xi_{0}(n)=0$ for $n \in\left[b_{1}, a_{1}\right],\left[b_{2}, a_{2}\right], \ldots,\left[b_{k}, a_{k}\right]$. Further, $\xi_{0}\left(b_{1}-1\right)=$ $\xi_{0}\left(a_{k}+1\right)=1$. If $\xi_{0}(n)=0$ for $r_{j}<n<\ell_{j+1}$ then we set $a_{j}=r_{j}, b_{j+1}=\ell_{j+1}$. Otherwise $a_{j}$ and $b_{j+1}$ are uniquely determined by the condition $\xi_{0}\left(a_{j}+\right.$ $1)=\xi_{0}\left(b_{j+1}-1\right)=1$. $T \xi_{0}$ is then defined as

$$
\begin{aligned}
T \xi_{0}\left(a_{j}\right) & =T \xi_{0}\left(b_{j}\right)=1, \quad 1 \leqslant j \leqslant k \\
T \xi_{0}(n) & =\xi_{0}(n), \quad n \in \mathbf{Z}-\left\{a_{1}, \ldots, a_{k}, b_{1}, \ldots, b_{k}\right\} .
\end{aligned}
$$


From the definition (3.8) for $f$ and (3.7) the inequality (3.15) follows. Note that $T$ is one-one whence (3.14) follows.

To prove (3.6) in the general case we can proceed as before and use (3.14). Thus the LHS of (3.6) is given by the sum,

$$
\begin{aligned}
& v\left\{\xi_{0}: \xi_{0}(n)=0, n \in \tilde{A}\right\}+\sum_{p=1}^{\infty} \sum_{\left\{j_{r} \in \tilde{A}, 1 \leqslant r \leqslant p: j_{1}<j_{2}<\cdots<j_{p}\right\}} v\left\{\xi_{0}: \xi\left(j_{r}\right)=1,\right. \\
& \left.1 \leqslant r \leqslant p, \xi_{0}(n)=0, n \in \tilde{A}-\left\{j_{1}, \ldots, j_{p}\right\}\right\} \\
& \times P\left(\zeta_{1}\left(j_{1}\right)=\zeta_{1}\left(j_{2}\right)=\cdots=\zeta_{1}\left(j_{p}\right)=0\right) .
\end{aligned}
$$

By arguing as in the proof of (3.11), (3.12), (3.13) we see that this sum is bounded by

$$
C^{k}\left[\frac{1}{1-C_{1} / \Lambda}+\left(\frac{C_{1}}{\Lambda}\right) \frac{1}{(\Lambda q)^{2}}\right]^{k} v\left\{\xi_{0}: \xi_{0}(n)=0, n \in \tilde{A}\right\},
$$

where $C, C_{1}$ are universal constants. The result follows now from (3.14).

The previous two lemmas imply a quantitative estimate on the solution of (1.3) which is global in time.

Corollary 3.1. Assume $A, \varepsilon, \eta$ are as in Proposition 2.1. Let $u(x, t)$ be a solution of (1.3) which has the property that $A v_{(2 n, 2 n+2)}[u(\cdot, 0)] \geqslant$ $\eta, n \in \mathbf{Z}$. Then there are universal constants $C \geqslant 1,0<\delta<1$, such that if $\sigma^{2}<\delta \eta$ there is the inequality,

$$
P\left(A v_{(0,2)}[u(\cdot, t)]<\eta\right) \leqslant \exp \left[-\eta / C \sigma^{2}\right], t \geqslant A
$$

Proof. From (3.1) and the maximum principle for (1.3) we have for any $m=1,2, \ldots$

$$
P\left(A v_{(0,2)}[u(\cdot, m A)]<\eta\right) \leqslant \int P\left(\sum_{n \in \mathbf{Z}} \xi_{m}(n) \zeta_{0}(n)=0\right) d v\left(\xi_{0}\right)
$$

where $v$ is any probability measure on initial configurations $\xi_{0}$ and $\zeta_{0}$ satisfies $\zeta_{0}(0)=1, \zeta_{0}(n)=0, n \neq 0$. If we choose $v$ to be the measure in Lemma 3.2 and use Lemma 3.1 we have from (3.17) the inequality,

$$
P\left(A v_{(0,2)}[u(\cdot, m A)]<\eta\right) \leqslant v\left\{\xi_{0}: \xi_{0}(0)=0\right\}
$$


whence (3.16) follows for $t=m A$. If $m A<t<(m+1) A$ we use the fact that we have already proven (3.16) for $t=(m-1) A$ and then we use Proposition 2.1.

Next we consider the Markov chain $\zeta_{m}$ with initial configuration $\zeta_{0}$ given by $\zeta_{0}(n)=1, n \leqslant 0, \zeta_{0}(n)=0, n>0$. For $m=1,2, \ldots$ we define $r_{m}$ by

$$
r_{m}=\sup \left\{n \in \mathbf{Z}: \zeta_{m}(n)=1\right\}
$$

It is evident that $-\infty<r_{m} \leqslant m$ with probability one. If we put $\alpha_{m}=$ $E\left[r_{m}\right]$ then it is also clear that for $k>m$ then $\alpha_{k} \leqslant \alpha_{m}+\alpha_{k-m}$. Hence $\alpha=$ $\lim _{m \rightarrow \infty} \alpha_{m} / m$ exists and $-\infty \leqslant \alpha \leqslant 1$.

Lemma 3.3. Suppose a probability measure $v$ exists satisfying (3.6). Then $\alpha \geqslant 0$ and $\lim _{m \rightarrow \infty} r_{m} / m=\alpha$ with probability 1 .

Proof. The fact that $\lim _{m \rightarrow \infty} r_{m} / m=\alpha$ with probability 1 follows from the subadditive ergodic theorem. ${ }^{(11)}$ Hence we are left to show that $\alpha \geqslant 0$ when (3.6) holds. We assume for contradiction that $\alpha<0$. Consider the Markov chain $\zeta_{m}$ with initial configuration given by $\zeta_{0}(n)=1, n \geqslant 0$, $\zeta_{0}(n)=0, n<0$. For $m=1,2, \ldots$ define $\ell_{m}$ by

$$
\ell_{m}=\inf \left\{n \in \mathbf{Z}: \zeta_{m}(n)=1\right\}
$$

By symmetry $\lim _{m \rightarrow \infty} \ell_{m} / m=-\alpha$ with probability 1 . Finally we consider $\zeta_{m}$ with initial configuration given by $\zeta_{0}(0)=1, \zeta_{0}(n)=0, n \neq 0$. Then it is clear that if $\zeta_{m} \neq \equiv$,

$$
\begin{aligned}
& r_{m} \geqslant \sup \left\{n \in \mathbf{Z}: \zeta_{m}(n)=1\right\}, \\
& \ell_{m} \leqslant \inf \left\{n \in \mathbf{Z}: \zeta_{m}(n)=1\right\} .
\end{aligned}
$$

Since we are assuming $\alpha<0$ it follows that $\lim _{m \rightarrow \infty} P\left(r_{m}<\ell_{m}\right)=1$, whence we conclude

$$
\lim _{m \rightarrow \infty} P\left(\zeta_{m}(n)=0, n \in \mathbf{Z}\right)=1
$$

From (3.6) we have that

$$
P\left(\zeta_{m}(n)=0, n \in \mathbf{Z}\right) \leqslant v\left\{\xi_{0}: \xi_{0}(0)=0\right\}=1-1 / \sum_{k=1}^{\infty} k f(k)<1 .
$$

Evidently this last inequality contradicts (3.19) whence we conclude that $\alpha \geqslant 0$. 
Lemma 3.4. Let $\zeta_{m}$ be the Markov chain defined by (3.2) with initial condition $\zeta_{0}$ satisfying $r_{0}<\infty$. Let $\zeta_{m}^{\prime}$ be the chain with initial conditions $\zeta_{0}^{\prime}$ satisfying $\zeta_{0}^{\prime}\left(r_{0}+1\right)=1$ and $\left\{n \in \mathbf{Z}: \zeta_{0}(n)=1\right\} \subset\left\{n \in \mathbf{Z}: \zeta_{0}^{\prime}(n)=1\right\}$. If $r_{m}^{\prime}=\sup \{n \in$ $\left.\mathbf{Z}: \zeta_{m}^{\prime}(n)=1\right\}$ then there is the inequality $E\left[r_{m}^{\prime}\right] \geqslant E\left[r_{m}\right]+1, m \geqslant 1$.

Proof. We argue as in Lemma 2.21 of Chapter VI of ref. 11. For a set $A \subset \mathbf{Z}$ we define sets $A_{m}, m \geqslant 0$, by $A_{0}=A$ and $A_{m}=\{n \in \mathbf{Z}$ : $\left.\zeta_{m}(n)=1\right\}$, where $\zeta_{m}$ is the Markov chain (3.2) with initial data $\zeta_{0}(n)=$ $0, n \notin A, \zeta_{0}(n)=1, n \in A$. Consider now a second subset $B \subset \mathbf{Z}$. Then the sets $A_{1}, B_{1}$ and $(A \cup B)_{1}$ are determined by the values of a collection of Bernoulli variables $X_{n}, n \in \mathbf{Z}$. It is clear that $(A \cup B)_{1}=A_{1} \cup B_{1}$, whence by induction one has $(A \cup B)_{m}=A_{m} \cup B_{m}, m \geqslant 1$. It follows that if for a set $A$ we put $r_{m}^{A}=\sup \left\{n: n \in A_{m}\right\}$ then

$$
r_{m}^{A \cup B}-r_{m}^{A}=\left(r_{m}^{B}-r_{m}^{A}\right)^{+}
$$

We put now $A=\left\{n \in \mathbf{Z}: \zeta_{0}(n)=1\right\}$ and $B=\left\{r_{0}+1\right\}$, whence $r_{m}=r_{m}, r_{m}^{\prime} \geqslant$ $r_{m}^{A \cup B}$. If $A^{\prime}=\left\{n \in \mathbf{Z}: n \leqslant r_{0}\right\}$ then it is clear that $r_{m}^{A^{\prime}} \geqslant r_{m}^{A}$. It follows now from (3.20) that

$$
r_{m}^{\prime}-r_{m} \geqslant\left(r_{m}^{B}-r_{m}^{A^{\prime}}\right)^{+}=r_{m}^{A^{\prime} \cup B}-r_{m}^{A^{\prime}}
$$

By translation invariance $E\left[r_{m}^{A^{\prime} \cup B}-r_{m}^{A^{\prime}}\right]=1$, whence the result follows.

Lemma 3.5. Suppose $q$ satisfies the conditions of Lemma 3.2 and $\alpha(q) \geqslant 0$ is the corresponding wave speed for the Markov chain $\zeta_{m}$ as given in Lemma 3.3. Then if $0<q^{\prime}<q$ there is the inequality $\alpha\left(q^{\prime}\right)-\alpha(q) \geqslant$ $q-q^{\prime}$.

Proof. Let $\zeta_{m}^{q}$ be the Markov chain $\zeta_{m}$ with parameter $q$. We can define the chains $\zeta_{m}^{q}$ and $\zeta_{m}^{q^{\prime}}$ on the same state space as follows: For $n \in \mathbf{Z}$ let $Y_{n}, Z_{n}$ be independent Bernoulli variables where $Y_{n}=0$ with probability $q$ and $Z_{n}=0$ with probability $q^{\prime} / q$. Then $\zeta_{m+1}^{q^{\prime}}$ is determined from $\zeta_{m}^{q}$ by taking the variables $X_{n}$ in (3.2) to be $X_{n}=Y_{n}$ while $\zeta_{m+1}^{q}$ is determined from $\zeta_{m}^{q^{\prime}}$ by putting $X_{n}=\min \left\{Y_{n}+Z_{n}, 1\right\}$.

We consider $\zeta_{m}^{q}$ and $\zeta_{m}^{q^{\prime}}$ with initial condition $\zeta_{m}^{q}(n)=\zeta_{m}^{q^{\prime}}(n)=1, n \leqslant 0$, $\zeta_{m}^{q}(n)=\zeta_{m}^{q^{\prime}}(n)=0, n>0$. It is evident that $\zeta_{m^{\prime}}^{q^{\prime}}(n) \geqslant \zeta_{m}^{q}(n), n \in \mathbf{Z}, m \geqslant 0$. Let $r_{m}^{q}$ and $r_{m}^{q^{\prime}}$ be defined as in (3.18). Then $r_{m}^{q^{\prime}} \geqslant r_{m}^{q}$. We define the stopping 
time $\tau$ to be $\tau=\inf \left\{m>0: r_{m}^{q^{\prime}}>r_{m}^{q}\right\}$. Evidently if $\tau<\infty$ then $r_{\tau}^{q^{\prime}} \geqslant r_{\tau}^{q}+1$. It follows then from Lemma 3.4 that

$$
E\left[r_{m}^{q^{\prime}} \mid \tau \leqslant m\right] \geqslant E\left[r_{m}^{q} \mid \tau \leqslant m\right]+1
$$

It is easy to see that

$$
P(\tau>m) \leqslant\left[1-q\left(1-q^{\prime} / q\right)\right]^{m}
$$

Putting the last two inequalities together we conclude

$$
\begin{aligned}
E\left[r_{m}^{q^{\prime}}\right] & \geqslant E\left[r_{m}^{q}\right]+1-\left[1-q\left(1-q^{\prime} / q\right)\right]^{m} \\
& =E\left[r_{m}^{q}\right]+m\left(q-q^{\prime}\right)+O\left(\left(q-q^{\prime}\right)^{2}\right) .
\end{aligned}
$$

Hence by a telescoping argument we have for any $0<q^{\prime}<q, m \geqslant 1$,

$$
E\left[r_{m}^{q^{\prime}}\right] \geqslant E\left[r_{m}^{q}\right]+m\left(q-q^{\prime}\right)
$$

whence the result follows.

We have already observed that $\alpha(q) \leqslant 1,0<q<1$. The next lemma shows that $\lim _{q \rightarrow 0} \alpha(q)=1$.

Lemma 3.6. There are universal constants $c_{1}, c_{2}>0$ such that if $0<q<c_{1}<1$ then

$$
\alpha(q) \geqslant 1+c_{2} / \log q
$$

Proof. We consider the chain $\zeta_{m}$ of (3.2) with initial data $\zeta_{0}(n)=1$, $n \leqslant 0, \zeta_{0}(n)=0, n>0$. Let $X_{n, m}, n, m \in \mathbf{Z}, m \geqslant 0$, be i.i.d. Bernoulli variables with $P\left(X_{n, m}=0\right)=q$. Then $\zeta_{m}(n)$ is determined by the values of the Bernoulli variables. For any $(n, m) \in \mathbf{Z}^{2}$ let $Q_{n, m}$ be the square centered at $(n, m)$ with side of length 1 . For integer $M \geqslant 1$ let $S_{M}$ be the set,

$$
S_{M}=\bigcup_{\left\{(n, m): 0 \leqslant m \leqslant M, \zeta_{m}(n)=1\right\}} Q_{n, m} .
$$

We define the region $\Omega_{M} \subset \mathbf{R}^{2}$ to be the connected component of the set $\left\{(x, y) \in \mathbf{R}^{2}: 0 \leqslant y \leqslant M, x \leqslant M+1\right\}-S_{M}$ which contains the point $(M+3 / 4,1 / 2)$. Evidently $\Omega_{M}$ is with probability 1 a finite region. The 
boundary $\partial \Omega_{M}$ of $\Omega_{M}$ contains the 3 line segments $\{(M+1, y): 0 \leqslant y \leqslant M\}$, $\{(x, 0): 1 / 2 \leqslant x \leqslant M+1\},\left\{(x, M): \rho_{M}+1 / 2 \leqslant x \leqslant M+1\right\}$, where $\rho_{M}$ is an integer satisfying $\rho_{M} \leqslant M$. The remainder of the boundary $\partial \Omega_{M}$ consists of sides of squares $Q_{n, m}$ with $\zeta_{m}(n)=1$. Let $A_{M}$ be the set of $(n, m) \in \mathbf{Z}^{2}$ with $\zeta_{m}(n)=1$ and such that a side of the square $Q_{n, m}$ is part of the boundary $\partial \Omega_{M}$. Let $B_{M} \subset A_{M}$ be those $(n, m)$ with $X_{n, m}=0$. We shall obtain inequalities relating $\left|A_{M}\right|,\left|B_{M}\right|, \rho_{M}$ and $M$.

We apply the divergence theorem in $\Omega_{M}$. Thus if $\mathbf{u}$ denotes the outward normal on $\partial \Omega_{M}$ we have that

$$
\int_{\partial \Omega_{M}}\left(\mathbf{e}_{1}+\mathbf{e}_{2}\right) \cdot \mathbf{u} d S=0,
$$

where $\mathbf{e}_{1}, \mathbf{e}_{2}$ are unit vectors in the $x$ and $y$ directions. Evaluating the integral on the line segments we conclude that,

$$
-\int_{\partial \Omega_{M} \cap S_{M}}\left(\mathbf{e}_{1}+\mathbf{e}_{2}\right) \cdot \mathbf{u} d S=M-\rho_{M}
$$

Observe that for the square $Q_{0,0}$ the half side $\left\{\left(\frac{1}{2}, y\right): 0 \leqslant y \leqslant \frac{1}{2}\right\}$ belongs to $\partial \Omega_{M}$. Similarly the half side $\left\{\left(\rho_{M}+\frac{1}{2}, y\right): M-\frac{1}{2} \leqslant y \leqslant M\right\}$ belongs to $\partial \Omega_{M}$. We include $(0,0)$ in the $A_{M}$, and in $B_{M}$ if $X_{0,0}=0$, but exclude $\left(\rho_{M}, M\right)$. Suppose now $(n, m) \in A_{M}-B_{M}$. Then $Q_{n, m}$ has a side in $\partial \Omega_{M}$ with normal parallel to $\mathbf{e}_{1}$. Further, if one moves along this side in the direction of $\mathbf{e}_{2}$ the next side of a square forming part of $\partial \Omega_{M}$ has normal $\mathbf{u}=\mathbf{e}_{2}$. Observe also that if the side of a square $Q_{n, m}$ belongs to $\partial \Omega_{M}$ and $\mathbf{u}=-\mathbf{e}_{2}$ on that side then $(n, m) \in B_{M}$. We conclude then from (3.21) that $\left|B_{M}\right| \geqslant$ $M-\rho_{M}$. If we apply the divergence theorem to the vector field $\mathbf{e}_{2}$ then we obtain the identity,

$$
\int_{\partial \Omega_{M} \cap S_{M}} \mathbf{e}_{2} \cdot \mathbf{u} d S=\rho_{M}
$$

This yields the inequality $\left|A_{M}-B_{M}\right| \leqslant\left|B_{M}\right|+\rho_{M}$, whence we have the two inequalities,

$$
\left|A_{M}\right| \leqslant 2\left|B_{M}\right|+M, \quad\left|B_{M}\right| \geqslant M-\rho_{M} .
$$

Let $\delta>0$ and consider the configurations such that $\rho_{M}<M(1-\delta)$. Letting $N=\left|B_{M}\right|$ we see from (3.22) that $N \geqslant M \delta$ and $\left|A_{M}\right| \leqslant 2 N+M$. Now the 
number of regions $\Omega_{M}$ satisfying $\left|A_{M}\right| \leqslant 2 N+M$ is bounded by $4^{2 N+M}$. Hence

$$
P\left(\rho_{M}<M(1-\delta)\right) \leqslant \sum_{N=M \delta}^{\infty} 4^{2 N+M} q^{N}
$$

It is clear that if $q$ satisfies $20 q<1$ and we take $\delta=-[1+\ln 4] /[2 \ln 4+$ $\ln q$ ], then there is the inequality,

$$
P\left(\rho_{M}<M(1-\delta)\right) \leqslant 5 e^{-M}
$$

The result follows from the Borel-Cantelli lemma.

Proposition 3.1. Assume $A, \varepsilon, \eta$ are as in Proposition 2.1. Let $c(\sigma)$ be the wave speed for the stochastic FKPP equation as given in Theorem 1.1. Then there are positive constants $C \geqslant 1, \delta<1$, such that if $\sigma^{2}<\delta \eta$ then $c(\sigma) \geqslant 2 / A-C \sigma^{2} / \eta$.

Proof. This follows from Lemmas 3.1 and 3.6. We put $\xi_{0}(n)=1, n \leqslant$ $0, \quad \xi_{0}(n)=0, n>0$. For any $\rho>0$ we define $\zeta_{0}$ by $\zeta_{0}(n)=1$ for $n \geqslant$ $[\alpha(q)-\rho] M, \zeta_{0}(n)=0, n<[\alpha(q)-\rho] M$, where $M$ is a positive integer. Let $\zeta_{m, M}, m \geqslant 0$, be the Markov chain with this initial data. Then by Lemma 3.3 we have that

$$
\lim _{M \rightarrow \infty} P\left(\sum_{n \in \mathbf{Z}} \xi_{0}(n) \zeta_{M, M}(n)=0\right)=0 .
$$

Hence from Lemma 3.1 we have that

$$
\lim _{M \rightarrow \infty} P\left(\xi_{M}(n)=0, \quad n \geqslant[\alpha(q)-\rho] M\right)=0
$$

Hence from (3.1) we have that $c(\sigma) \geqslant 2[\alpha(q)-\rho] / A$. The result follows from Lemma 3.6 on letting $\rho \rightarrow 0$.

\section{CONTINUITY OF THE WAVE SPEED}

In this section we prove the continuity of the wave speed $c(\sigma)$ at $\sigma=0$ by establishing the lower bound for inequality (1.8) of Theorem 1.2. We have already observed that $c(0)=2$ and that $c(\sigma) \leqslant c(0)$. Hence to prove continuity we need to find a lower bound on $c(\sigma)$ which increases to 2 as $\sigma \rightarrow 0$. Our initial goal will be to obtain a refinement of Proposition 2.1. Towards that we consider the deterministic case $\sigma=0$. 
Lemma 4.1. Let $\eta>0, A>2$, and $u(x, t)$ be the solution of (1.3) for $\sigma=0$ with initial condition $u(x, 0)$. Suppose $A v_{(-1,1)}[u(\cdot, 0)] \geqslant \eta$. Then there are universal positive constants $C_{1}, C_{2}$ such that $u(x, A) \geqslant \eta$ for $x$ satisfying

$$
|x| \leqslant 2 A\left[1-\eta e^{C_{1} \sqrt{A}}\right]-C_{2} \log A .
$$

Proof. We may assume wlog that $u(x, 0)=u_{0}(x)$ where $u_{0}(x)=0$, $|x|>1$, and $A v_{(-1,1)}\left[u_{0}\right]=\eta$. Then $u(x, t)$ is given by the Feymann-Kac formula,

$$
u(x, t)=E_{x}\left[\exp \left\{\int_{0}^{t} 1-u(B(s), t-s) d s\right\} u_{0}(B(t))\right],
$$

where $B(t)$ is Brownian motion with $B(0)=x$ and $E_{x}\left[\{B(t)-B(0)\}^{2}\right]=2 t$. Evidently we have that

$$
u(x, t) \leqslant e^{t} \int_{-1}^{1} G(x-y, t) u_{0}(y) d y,
$$

where $G$ is given by (2.1). Hence there is a universal constant $C$ such that

$$
u(x, t) \leqslant C \eta \exp \left[\frac{-x^{2}}{4 t}+t\right], \quad|x| \leqslant 2 t, \quad t \geqslant 1 .
$$

We can rewrite (4.2) as

$$
u(x, t)=\int G(x-y, t) H(x, y, t) u_{0}(y) d y,
$$

where

$$
H(x, y, t)=E\left[\exp \left\{\int_{0}^{t} 1-u(B(s), t-s) d s\right\} \mid B(0)=x, B(t)=y\right] .
$$

It is well known that Brownian motion $B(s), 0 \leqslant s \leqslant t$, conditioned on $B(0)=x, B(t)=y$ has the same law as $B^{\prime}(s)-\frac{s}{t} B^{\prime}(t)+\left(1-\frac{s}{t}\right) x+\frac{s}{t} y$, where $B^{\prime}(s), s>0$, is Brownian motion with $B^{\prime}(0)=0, E\left[B^{\prime}(s)^{2}\right]=2 s$. Now there is a universal constant $c_{1}>0$ such that,

$$
P\left(\sup _{0<s<t}\left|B^{\prime}(s)-\frac{s}{t} B^{\prime}(t)\right|<\sqrt{t}\right)>c_{1} .
$$


If we write the conditional Brownian motion $B(s)=\left(1-\frac{s}{t}\right) x+z$, it is clear from (4.3) that

$$
u(B(s), t-s) \leqslant C \eta \exp \left[(t-s)\left\{1-\frac{x^{2}}{4 t^{2}}\right\}-\frac{z x}{2 t}-\frac{z^{2}}{4(t-s)}\right] .
$$

Thus if $2 t-\sqrt{t} \leqslant|x| \leqslant 2 t, \quad|y| \leqslant 1,\left|B^{\prime}(s)-\frac{s}{t} B^{\prime}(t)\right|<\sqrt{t}$, there is the inequality,

$$
u(B(s), t-s) \leqslant C^{\prime} \eta e^{2 \sqrt{t}}, \quad 0 \leqslant s \leqslant t-1
$$

for some universal constant $C^{\prime}$. It follows now from (4.5), (4.6) that

$$
\begin{aligned}
H(x, y, t) \geqslant & c_{1} \exp \left[(t-1)\left\{1-C^{\prime} \eta e^{2 \sqrt{t}}\right\}\right], \\
& 2 t-\sqrt{t} \leqslant|x| \leqslant 2 t,|y| \leqslant 1, \quad t>1 .
\end{aligned}
$$

From this last inequality and (4.4) we conclude,

$$
\begin{aligned}
& u(x, t) \geqslant \frac{2 c_{1} e^{-3}}{\sqrt{4 \pi}} \eta \exp \left[t\left\{1-C^{\prime} \eta e^{2 \sqrt{t}}\right\}-\frac{1}{2} \log t-\frac{x^{2}}{4 t}\right], \\
& 2 t-\sqrt{t} \leqslant|x| \leqslant 2 t, \quad t>1 .
\end{aligned}
$$

Let us assume now that $\eta e^{3 \sqrt{t}} \leqslant 1$. Then from the previous inequality we see that there is a universal constant $C_{3}>1$ such that $u(x, t) \geqslant \eta$ provided $(x, t)$ lies in the region,

$$
2 t-\sqrt{t} \leqslant|x| \leqslant 2 t\left[1-C^{\prime} \eta e^{2 \sqrt{t}}\right]-\log t, t \geqslant C_{3} .
$$

To complete the proof of the lemma it is sufficient to show that for any $L>1$ there exists $T(L)>1$, depending only on $L$, such that

$$
u(x, t) \geqslant \eta, \quad|x| \leqslant L, \quad t \geqslant T(L) .
$$

The inequality (4.8) follows from Lemma 2.1. It is clear now that (4.1) with $C_{1}=3, C_{2}=1$ is a consequence of (4.7), (4.8). 
We consider the stochastic equation

$$
u_{t}=u_{x x}+2 u_{x}+u(1-u)+\sigma \sqrt{u(1-u)} W
$$

with initial data $u(x, 0)$ satisfying $u(x, 0)=0,|x|>1$. We shall be interested in solutions of (4.9) on intervals $[-L, L], L \geqslant 2$, with Dirichlet boundary conditions. Let $G_{L}$ be the Dirichlet Green's function for the equation $u_{t}=$ $u_{x x}+2 u_{x}$ on the interval $[-L, L]$. Evidently there is the inequality,

$$
G_{L}(x, y, t) \leqslant \frac{1}{\sqrt{4 \pi t}} \exp \left[-\frac{1}{4 t}(x-y+2 t)^{2}\right], \quad t>0 .
$$

Define $N_{L}(x, t)$ by

$$
N_{L}(x, t)=\int_{-L}^{L} d y \int_{0}^{t} d s e^{(t-s)} G_{L}(x, y, t-s) \sqrt{u(y, s)[1-u(y, s)]} W(y, s),
$$

where $u$ is the solution to (4.9). Then we have the following generalization of Lemma 2.2:

Lemma 4.2. Let $L, \alpha \geqslant 2$. There is a universal constant $C>0$ such that,

$$
\begin{gathered}
P\left(\sup _{|x| \leqslant L, 0 \leqslant t \leqslant L^{2}}\left|N_{L}(x, t)\right|>\alpha e^{2 L} \sqrt{A v_{(-1,1)}[u(\cdot, 0)]}\right) \\
\leqslant C L \exp \left[-\frac{1}{C} \min \left\{\alpha^{2}, \alpha \sqrt{A v_{(-1,1)}[u(\cdot, 0)]} / \sigma L\right\}\right] .
\end{gathered}
$$

Proof. We proceed as in Lemma 2.2. Thus we need to estimate $\left\langle\exp \left[2 \theta^{2} V_{L}(x, t)\right]\right\rangle$ where

$$
V_{L}(x, t)=\int_{-L}^{L} d y \int_{0}^{t} d s e^{2(t-s)} G_{L}^{2}(x, y, t-s) u(y, s) .
$$

This expectation only increases if we assume $u(x, t)$ is the solution to the equation,

$$
u_{t}=u_{x x}+2 u_{x}+u+\sigma \sqrt{u} W
$$


on $[-L, L]$ with Dirichlet boundary conditions and the same initial data as in (4.9). Let $\varphi:[-L, L] \times(0, \infty) \rightarrow \mathbf{R}$ be non-negative and consider the variable $V_{\varphi}(t)$,

$$
V_{\varphi}(t)=\int_{-L}^{L} d y \int_{0}^{t} d s \varphi(y, s) u(y, s),
$$

where $u(x, t)$ is the solution to $(4.13)$ on $[-L, L]$ with Dirichlet boundary conditions and initial data $u(\cdot, 0)$ with support in $[-1,1]$. Then

$$
\left\langle\exp \left[V_{\varphi}(t)\right]\right\rangle=\exp \left[\int_{-L}^{L} u(x, 0) v(x, 0) d x\right]
$$

where $v(x, s), s \leqslant t,|x|<L$, satisfies the initial-boundary value problem,

$$
\begin{gathered}
\frac{\partial v}{\partial s}+v_{x x}-2 v_{x}+v+\frac{\sigma^{2}}{2} v^{2}+\varphi=0, \quad s<t, \\
v(x, t)=0, \quad|x|<L, \quad v(x, s)=0, \quad|x|=L, \quad s<t .
\end{gathered}
$$

Letting, $X(s)$ be Brownian motion with drift -2 in $[-L, L]$ and killed on the boundary then the solution $v(x, s)$ of (4.14) is a fixed point of the mapping $T$ of (2.9). Suppose now that $\varphi$ satisfies for some $x$ with $|x|<L$ the inequality,

$$
|\varphi(y, s)| \leqslant \frac{e^{(t-s)}}{\beta \sqrt{t-s}} G_{L}(x, y, t-s), \quad|y|<L, \quad 0 \leqslant s \leqslant t,
$$

where $\beta>0$ is a constant. For a function $v:[-L, L] \times[0, t] \rightarrow \mathbf{R}$ let

$$
\|v\|=\sup _{\left|x^{\prime}\right| \leqslant L, 0 \leqslant s \leqslant t}\left|v\left(x^{\prime}, s\right)\right| .
$$

Then we have from (2.9) that

$$
\begin{aligned}
\left|T v\left(x^{\prime}, s\right)\right| \leqslant & \exp \left[\frac{\sigma^{2}}{2}\|v\|(t-s)\right] \int_{s}^{t} d s^{\prime} e^{s^{\prime}-s} \int_{-L}^{L} d y \\
& \times G_{L}\left(y, x^{\prime}, s^{\prime}-s\right) \frac{e^{t-s^{\prime}}}{\beta \sqrt{t-s^{\prime}}} G_{L}\left(x, y, t-s^{\prime}\right) \\
= & \exp \left[\frac{\sigma^{2}}{2}\|v\|(t-s)\right] e^{t-s} G_{L}\left(x, x^{\prime}, t-s\right) \frac{2 \sqrt{t-s}}{\beta} .
\end{aligned}
$$


From (4.10) we see that

$$
e^{t-s} G_{L}\left(x, x^{\prime}, t-s\right) 2 \sqrt{t-s} \leqslant \frac{1}{\sqrt{\pi}} e^{2 L}, \quad|x|,\left|x^{\prime}\right| \leqslant L,
$$

whence we conclude that

$$
\|T v\| \leqslant \frac{e^{2 L}}{\beta \sqrt{\pi}} \exp \left[\frac{\sigma^{2}}{2}\|v\| t\right] .
$$

Now let $\mathcal{S}_{L, \beta}=\left\{v:[-L, L] \times[0, t] \rightarrow \mathbf{R}:\|v\| \leqslant e^{2 L} / \beta\right\}$. Then if $\beta$ satisfies $\beta \geqslant$ $\sigma^{2} e^{2 L} t$ it follows that $T$ maps $\mathcal{S}_{L, \beta}$ into $\mathcal{S}_{L, \beta}$. In a similar way to the derivation of (4.18) one can see that $T$ is also a contraction mapping on $\mathcal{S}_{L, \beta}$ whence there is a unique solution $v \in \mathcal{S}_{L, \beta}$ to the equation $v=T v$. We conclude that if $\varphi$ satisfies (4.16) then

$$
\left\langle\exp \left[V_{\varphi}(t)\right]\right\rangle \leqslant \exp \left[2 e^{2 L} A v_{(-1,1)}[u(\cdot, 0)] / \beta\right]
$$

provided $\beta \geqslant \sigma^{2} e^{2 L} t$. We apply (4.19) to (4.12). Thus $2 \theta^{2} V_{L}(x, t)=V_{\varphi}(t)$ where we can take $\beta=\sqrt{\pi} e^{-2 L} / \theta^{2}$ from (4.17). We have therefore the inequality,

$$
\left\langle\exp \left[2 \theta^{2} V_{L}(x, t)\right]\right\rangle \leqslant \exp \left[2 \theta^{2} e^{4 L} A v_{(-1,1)}[u(\cdot, 0)] / \sqrt{\pi}\right],
$$

provided $\theta^{2} e^{4 L} t \leqslant \sqrt{\pi} / \sigma^{2}$. From (4.20) we see that

$$
\begin{aligned}
& P\left(\left|N_{L}(x, t)\right|>\alpha e^{2 L} \sqrt{A v_{(-1,1)}[u(\cdot, 0)]}\right) \\
& \leqslant 2 \exp \left[-\frac{1}{8} \min \left\{\alpha^{2}, \alpha \sqrt{A v_{(-1,1)}[u(\cdot, 0)]} / \sigma \sqrt{t}\right\}\right]
\end{aligned}
$$

provided $|x| \leqslant L, t>0$.

Next we obtain an estimate on the difference $N_{L}(x, t)-N_{L}(x, t-\delta)$ where $0<\delta<t / 3$. To do this we need to estimate the LHS of (4.14) where $\varphi(y, s)$ is given by

$$
\begin{gathered}
\varphi(y, s)=2 \theta^{2} e^{2(t-s)} G_{L}^{2}(x, y, t-s), \quad|y|<L, \quad t-\delta<s<t, \\
\varphi(y, s)=2 \theta^{2}\left\{e^{(t-s)} G_{L}(x, y, t-s)-e^{t-\delta-s} G_{L}(x, y, t-\delta-s)\right\}^{2} \\
|y|<L, \quad 0<s<t-\delta .
\end{gathered}
$$


Proceeding as before we define for a function $v:[-L, L] \times[0, t] \rightarrow \mathbf{R}$ the norm $\|v\|_{\delta}$ as

$$
\|v\|_{\delta}=\sup _{|x| \leqslant L, 0 \leqslant s \leqslant t}(t+\delta-s)^{1 / 2}|v(x, s)|
$$

If we use the inequality

$$
\left\{\frac{\partial}{\partial s} e^{(t-s)} G_{L}(x, y, t-s)\right\}^{2} \leqslant \frac{C e^{2 L}}{(t-s)^{5 / 2}} e^{t-s} G_{L}(x, y, t-s)
$$

for some universal constant $C$, it follows that with $\varphi$ as in (4.22) then there is the inequality,

$$
\|T v\|_{\delta} \leqslant C_{1} \theta^{2} \delta^{1 / 2} e^{4 L} \exp \left[C_{1} \sigma^{2}\|v\|_{\delta} \sqrt{t}\right]
$$

for some universal constant $C_{1}$. Let $\mathcal{S}_{\theta, \delta}$ be the set

$$
\mathcal{S}_{\theta, \delta}=\left\{v:[-L, L] \times[0, t] \rightarrow \mathbf{R}:\|v\|_{\delta} \leqslant 2 C_{1} \theta^{2} \delta^{1 / 2} e^{4 L}\right\}
$$

If $C_{1}^{2} \theta^{2} \sigma^{2} e^{4 L}(t \delta)^{1 / 2} \leqslant 1 / 4$ then $T$ maps $\mathcal{S}_{\theta, \delta}$ to $\mathcal{S}_{\theta, \delta}$ and one can easily see that $T$ is a contraction. We conclude then that,

$$
\begin{aligned}
& \left\langle\exp \left[\theta\left\{N_{L}(x, t)-N_{L}(x, t-\delta)\right\}\right]\right\rangle \\
& \quad \leqslant \exp \left[4 C_{1} \theta^{2}(\delta / t)^{1 / 2} e^{4 L} A v_{(-1,1)}[u(\cdot, 0)]\right] .
\end{aligned}
$$

Arguing as before we conclude that

$$
\begin{aligned}
& P\left(\left|N_{L}(x, t)-N_{L}(x, t-\delta)\right|>\alpha e^{2 L} \sqrt{A v_{(-1,1)}[u(\cdot, 0)]}\right) \\
& \quad \leqslant 2 \exp \left[-\frac{1}{32 C_{1}} \min \left\{\frac{\alpha^{2}}{(\delta / t)^{1 / 2}}, \frac{\alpha \sqrt{A v_{(-1,1)}[u(\cdot, 0)]}}{(\delta / t)^{1 / 4} \sigma \sqrt{t}}\right\}\right] .
\end{aligned}
$$

Finally we need to obtain an estimate on the difference $N_{L}(x+$ $\left.\delta^{1 / 2}, t\right)-N(x, t)$. To do this we use the inequality,

$$
\left\{\frac{\partial}{\partial x} e^{(t-s)} G_{L}(x, y, t-s)\right\}^{2} \leqslant \frac{C e^{2 L}}{\sqrt{t-s}}\left[1+\frac{1}{(t-s)}\right] e^{t-s} G_{L}(x, y, t-s)
$$


for some universal constant $C$. If $0<\delta<t / 3<1$ then we can argue as in Lemma 2.2 to obtain an estimate similar to (4.23),

$$
\begin{array}{r}
P\left(\left|N_{L}\left(x+\delta^{1 / 2}, t\right)-N_{L}(x, t)\right|>\alpha e^{2 L} \sqrt{A v_{(-1,1)}[u(\cdot, 0)]}\right) \\
\leqslant 2 \exp \left[-\frac{1}{C_{1}} \min \left\{\frac{\alpha^{2}}{(\delta / t)^{1 / 2}}, \frac{\alpha \sqrt{A v_{(-1,1)}[u(\cdot, 0)]}}{(\delta / t)^{1 / 4} \sigma \sqrt{t}}\right\}\right]
\end{array}
$$

for some universal constant $C_{1}$. Suppose now $0<\delta<1, t>3$. Then in the same way we derived (4.21) we have the inequality,

$$
\begin{aligned}
& P\left(\left|N_{L}\left(x+\delta^{1 / 2}, t\right)-N_{L}(x, t)\right|>\alpha e^{2 L} \sqrt{A v_{(-1,1)}[u(\cdot, 0)]}\right) \\
& \quad \leqslant 2 \exp \left[-\frac{1}{C_{1}} \min \left\{\frac{\alpha^{2}}{\delta+(\delta / t)^{1 / 2}}, \frac{\alpha \sqrt{A v_{(-1,1)}[u(\cdot, 0)]}}{\left[\delta+(\delta / t)^{1 / 2}\right]^{1 / 2} \sigma \sqrt{t}}\right\}\right] .
\end{aligned}
$$

The result follows from the inequalities (4.21), (4.23), (4.24), (4.25).

Lemma 4.3. There is a universal constant $c>0$ such that for $|x|,|y|<L / 2$ there is the inequality,

$$
e^{L^{2}} G_{L}\left(x, y, L^{2}\right) \geqslant \frac{c}{L} e^{y-x}
$$

Proof. This follows from the representation of conditional Brownian motion given in Lemma 4.1 and the inequality (4.6).

Lemma 4.4. Let $u(x, t)$ be a solution of (4.9) on the interval $[-L, L]$ with Dirichlet boundary conditions and initial data $u(x, 0)$ satisfying $A v_{(-1,1)}[u(\cdot, 0)] \geqslant \eta$. Then there are universal constants $c_{1}, C_{2}, C_{3}>0$, such that for $|x|<L / 2$, there is the inequality,

$$
u\left(x, L^{2}\right) \geqslant c_{1} \eta \exp \left[-x-C_{2} \eta L^{2} e^{2 L}-\log L\right]-\eta
$$

with probability at least

$$
1-C_{3} L \exp \left[\frac{-\eta}{C_{3} \sigma^{2} e^{4 L}}\right]
$$


Proof. We proceed as in the proof of Proposition 2.1. First we assume wlog that $u(x, 0)=0$ for $|x|>1$ and $A v_{[-1,1]} u(\cdot, 0)=\eta$. We apply Lemma 4.2 with $\alpha=\sqrt{\eta} e^{-2 L} / \sigma$. It follows that

$$
\sup _{|x| \leqslant L, 0 \leqslant t \leqslant L^{2}} N_{L}(x, t) \leqslant \eta / \sigma
$$

with probability (4.27). If we apply the analogue of (2.20) with $\gamma=1$ we obtain the inequality,

$$
\sup _{|x| \leqslant L, 1 \leqslant t \leqslant L^{2}} u(x, t) \leqslant C_{2} \eta e^{2 L}
$$

with probability at least (4.27). The result follows by applying the analogue of (2.20) again, this time with $\gamma=1-C_{2} \eta e^{2 L}$ and using Lemma 4.3.

Proof of Theorem 1.2, inequality (1.8). In Lemma 4.4 we take $\eta=$ $\sigma, L=\frac{1}{8}|\log \sigma|$. With this choice it follows that if $\sigma$ is sufficiently small and $x \in[-\log L-1,-\log L+1]$ then $u\left(x, L^{2}\right) \geqslant \eta$ with probability at least $1-\exp \left[-1 / C_{3} \sqrt{\sigma}\right]$.

To finish the proof we proceed as in section 3. Thus in analogy to (3.1) we define $\xi_{m}$ for a solution $u(x, t)$ of (1.3) by,

$$
\begin{array}{ll}
\xi_{m}(n)=1 & \text { if } A v_{\left(n\left(2 L^{2}-2 \log L\right)-1, n\left(2 L^{2}-2 \log L\right)+1\right)}\left[u\left(\cdot, m L^{2}\right)\right] \geqslant \eta, \\
\xi_{m}(n)=0 & \text { if } A v_{\left(n\left(2 L^{2}-2 \log L\right)-1, n\left(2 L^{2}-2 \log L\right)+1\right)}\left[u\left(\cdot, m L^{2}\right)\right]<\eta .
\end{array}
$$

If we define $\zeta_{m}$ as in (3.2) with $q=\exp \left[-1 / C_{3} \sqrt{\sigma}\right]$ then the results of Section 3 apply to $\xi_{m}$ and $\zeta_{m}$. If the initial data for $u(x, t)$ is $u(x, 0)=1, x<$ $0, u(x, 0)=0, x>0$, then it follows in particular from Lemma 3.6 that,

$$
\liminf _{m \rightarrow \infty}\left[\sup \left\{n \in \mathbf{Z}: \xi_{m}(n)=1\right\} / m\right] \geqslant 1-C \sqrt{\sigma}
$$

for some universal constant $C>0$. The result follows now from (4.29).

\section{EXISTENCE OF WAVE SPEED}

We first show that the limit (1.6) of Theorem 1.2 exists as $t \rightarrow \infty$ through the integers. 
Lemma 5.1. Let $u$ be a solution of (1.3) such that $-\infty<a(0) \leqslant$ $b(0)<\infty$. Then there is a constant $c(\sigma) \geqslant-\infty$ depending only on $\sigma$ such that,

$$
\lim _{m \rightarrow \infty} \frac{b(m)}{m}=c(\sigma)
$$

with probability 1 , as $m \in \mathbf{Z}$ goes to infinity.

Proof. We first consider the case when $a(0)=b(0)$, whence we may assume $u(x, 0)=1, x<0$ and $u(x, 0)=0, x>0$. For $m=0,1,2, \ldots$, we define the random variable $X_{0, m}$ by $X_{0, m}=b(m)$. We also define random variables $X_{r, m}, 1 \leqslant r \leqslant m$. To do this let $u(x, t), t \geqslant r$, be the solution of (1.3) with initial condition $u(x, r)=1, x \leqslant b(r), u(x, r)=0, x>b(r)$. Then we put

$$
X_{r, m}=\sup \{x \in \mathbf{R}: u(x, m)>0\}-b(r)
$$

Evidently one has that $X_{0, m} \leqslant X_{0, r}+X_{r, m}, 0 \leqslant r \leqslant m$. It is also clear that for each $k \geqslant 1$ the variables $X_{(m-1) k, m k}, m \geqslant 1$, are independent identically distributed. Further, for each $m \geqslant 0$ the set of variables $\left\{X_{m, m+k}: k \geqslant\right.$ $0\}$ have the same distribution as $\left\{X_{m+1, m+k+1}: k \geqslant 0\right\}$. Finally one notes from $^{(13)}$ that $E\left[X_{0,1}^{+}\right]<\infty$. The result follows now from the subadditive ergodic theorem, Theorem 2.6 of Chapter VI of ref. 11. To generalize to the case $b(0)-a(0)>0$, let $b_{H}(m)$ denote the values of $b(m)$ for the Heaviside initial data just considered. It follows now from ref. 18 that

$$
b_{H}(m)+a(0) \leqslant b(m) \leqslant b_{H}(m)+b(0), m=1,2, \ldots,
$$

whence the result for Heaviside initial data implies the general result.

To extend the limit in Lemma 5.1 to all $t \rightarrow \infty$ we use a result of ref. 20 which directly applies to the situation here.

Lemma 5.2. Let $0<t<1, a>0$. Then there is a constant $C(\sigma)$ depending only on $\sigma$, such that

$$
P\left[\sup _{0<s<t}\{b(s)-b(0)\}>a\right] \leqslant \frac{C(\sigma)}{\sqrt{t}} \exp \left[\frac{-a^{2}}{8 t}\right]
$$

Proof. Same as for Proposition 3.2 of ref. 20. 
Lemma 5.3. Let $u$ be a solution of (1.3) such that $-\infty<a(0) \leqslant$ $b(0)<\infty$. Then there is a constant $c(\sigma) \geqslant-\infty$ depending only on $\sigma$ such that,

$$
\lim _{t \rightarrow \infty} \frac{b(t)}{t}=c(\sigma)
$$

with probability 1 .

Proof. It follows from Lemma 5.2 that for $m=1,2, \ldots$, there is the inequality,

$$
\begin{aligned}
& P\left[\sup _{0 \leqslant s \leqslant 1}\{b(s+m)-b(m), b(m+1)-b(s+m)\}>\sqrt{m}\right] \\
& \quad \leqslant C(\sigma) \exp [-m / 8] .
\end{aligned}
$$

Hence the result follows from the Borel-Cantelli lemma.

Lemma 5.4. Let $c(\sigma)$ be the wave speed as defined in Lemma 5.3. Then $c(\sigma) \geqslant 0$.

Proof. We can assume wlog that $a(0)=0$, whence

$$
<u(x, t)>\geqslant \int_{-\infty}^{0} G(x-y, t) d y .
$$

Thus for $x<0$,

$$
\begin{aligned}
<1-u(x, t)> & \leqslant \int_{|x|}^{\infty} \frac{1}{\sqrt{4 \pi t}} \exp \left[\frac{-z^{2}}{4 t}\right] d z \\
& \leqslant \frac{1}{|x|} \sqrt{\frac{t}{\pi}} \exp \left[\frac{-x^{2}}{4 t}\right] .
\end{aligned}
$$

It follows that

$$
\sum_{m=1}^{\infty} P\left(u\left(-m^{2 / 3}, m\right)<\frac{1}{2}\right)<\infty .
$$

We conclude from Borel-Cantelli that

$$
\liminf _{m \rightarrow \infty} u\left(-m^{2 / 3}, m\right) \geqslant \frac{1}{2}
$$

with probability 1 . The result follows. 
Next we wish to show that $c(\sigma)>0$. First we show that this is the case for a discrete version of (1.3). Proceeding as in ref. 5 we discretize the $x$ axis with lattice spacing $h$ to obtain the system of equations,

$$
\begin{aligned}
\frac{\partial u(x, t)}{\partial t}= & \frac{D}{h^{2}}[u(x+h, t)-2 u(x, t)+u(x-h, t)] \\
& +\gamma u(x, t)[1-u(x, t)]+\frac{\sigma}{\sqrt{h}} \sqrt{u(x, t)[1-u(x, t)]} W(x, t),
\end{aligned}
$$

where the $W(x, t), \quad x \in h \mathbf{Z}$, are independent copies of white noise. It was shown in ref. 5 that expectations of polynomials in the variables $u(x, t), \quad x \in h \mathbf{Z}$, are equal to expectations for a dual process $\mathbf{N}(t): \mathbf{Z} \rightarrow$ $\mathbf{Z}^{+}$where $\mathbf{Z}^{+}$denotes the non-negative integers. Let $\mathbf{N}(t)=\left(\ldots, N_{i-1}(t)\right.$, $\left.N_{i}(t), N_{i+1}(t), \ldots\right)$, where $N_{i}(t)$ is the number of particles at site $i \in \mathbf{Z}$. The Markov chain $\mathbf{N}(t)$ is defined by the transition rates at which particles are born, coagulate and diffuse. Birth, for which $A \rightarrow A+A$, occurs at rate $\gamma$. Coagulation, for which $A+A \rightarrow A$, occurs at rate $\sigma^{2} / h$. A particle diffuses from $i \in \mathbf{Z}$ to $i+1$ at rate $D / h^{2}$ and also from $i$ to $i-1$ at rate $D / h^{2}$. Let $u(x, t)$ be the solution of $(5.1)$ with initial data $u(x, 0)=1, x \leqslant 0$, $u(x, 0)=0, x>0$. Then from ref. 5 we have the identity,

$$
\begin{aligned}
& <u(x, t)> \\
& =P\left[N_{j}(t)>0 \text { for some } j \leqslant 0 \mid N_{i}(0)=0, i \neq x / h, N_{x / h}(0)=1\right] .
\end{aligned}
$$

We can bound from below the probability on the RHS of (5.2) by the probability for a Markov chain on $h \mathbf{Z} \times\{1,2\}$. The transitions in the chain occur at exponential rates as follows:

$$
\begin{aligned}
& (x, 2) \rightarrow(x, 1) \text { at rate } \sigma^{2} / h+2 D / h^{2}, \\
& (x, 2) \rightarrow(x-h, 1) \text { at rate } 2 D / h^{2}, \\
& (x, 1) \rightarrow(x, 2) \text { at rate } \gamma, \\
& (x, 1) \rightarrow(x+h, 1) \text { at rate } D / h^{2}, \\
& (x, 1) \rightarrow(x-h, 1) \text { at rate } D / h^{2} .
\end{aligned}
$$

Let $[X(t), s(t)]$ with $X(t) \in h \mathbf{Z}, s(t) \in\{1,2\}$ be the position at time $t$ of the Markov chain with these transition rates. 
Lemma 5.5. There is the inequality,

$$
\begin{aligned}
P[X(t) \leqslant 0 \mid & X(0)=x, \quad s(0)=1] \leqslant P\left[N_{j}(t)>0 \quad \text { for some } j \leqslant 0 \mid N_{i}(0)=0,\right. \\
& \left.i \neq x / h, \quad N_{x / h}(0)=1\right] .
\end{aligned}
$$

Proof. We do this by a coupling argument. Consider the initial state $\mathbf{N}(0)$ of the Markov chain $\mathbf{N}(t)$ to consist of 1 red particle at site $x / h \in \mathbf{Z}$. The state at time $t$ consists of black particles and 1 red particle or black particles and 2 red particles on the same site. The transitions between black and red particles are as follows:

(a) Black particles give birth to black particles, and 2 black particles coagulate to 1 black particle.

(b) A black particle and a red particle coagulate to 1 red particle, while 2 red particles coagulate to 1 red particle.

(c) If there is just 1 red particle it gives birth to a second red particle. If there are 2 red particles on the same site they give birth to black particles.

(d) Suppose there are 2 red particles at $i \in \mathbf{Z}$. If one of the red particles moves to $i+1$ it becomes black. If one of the red particles moves to $i-1$ it remains red but the red particle left at site $i$ now becomes black.

Let $X(t) / h \in \mathbf{Z}$ be the position of the red particles at time $t$ and $s(t)$ be the number. Thus $X(t) \in h \mathbf{Z}, s(t) \in\{1,2\}, X(0)=x, s(0)=1$. It is clear that $[X(t), s(t)] \in h \mathbf{Z} \times\{1,2\}$ has the same law as the Markov chain we already defined, whence the result.

Lemma 5.6. Suppose $x>0, t>0$ and put $\rho=2 D \gamma t /\left[\sigma^{2}+\gamma h+\right.$ $4 D / h]-x$. There is a constant $C(D, \gamma, h)>0$ depending only on $D, \gamma, h$, such that if $\rho>0$ there is the inequality,

$$
P(X(t)>0 \mid X(0)=x, s(0)=1) \leqslant \frac{C(D, \gamma, h)}{\sqrt{t}} \exp \left[-\frac{\rho^{2}}{C(D, \gamma, h) t}\right] .
$$

Proof. Let $f: h \mathbf{Z} \times\{1,2\} \rightarrow \mathbf{R}$ be a bounded function and $v(x, s, t)$ be given for $x \in h \mathbf{Z}, s \in\{1,2\}, t>0$ by

$$
v(x, s, t)=E[f(X(t), s(t)) \mid X(0)=x, s(0)=s] .
$$


Then $v(x, s, t)$ is a solution to the equations,

$$
\begin{aligned}
& \frac{\partial v}{\partial t}(x, 1, t)= \frac{D}{h^{2}}[v(x+h, 1, t)-2 v(x, 1, t)+v(x-h, 1, t)] \\
&+\gamma[v(x, 2, t)-v(x, 1, t)] \\
& \frac{\partial v}{\partial t}(x, 2, t)= \frac{2 D}{h^{2}}[v(x-h, 1, t)-v(x, 2, t)] \\
&+\left(\frac{\sigma^{2}}{h}+\frac{2 D}{h^{2}}\right)[v(x, 1, t)-v(x, 2, t)]
\end{aligned}
$$

with initial condition $v(x, s, 0)=f(x, s)$. Since the equations are translation invariant we can solve them by going to Fourier variables. Thus for $\xi \in[-\pi / h, \pi / h]$ we define $\hat{v}(\xi, s, t)$ by

$$
\hat{v}(\xi, s, t)=h \sum_{x \in h \mathbf{Z}} v(x, s, t) e^{i x \xi}
$$

Then we have that

$$
\frac{\partial}{\partial t}\left[\begin{array}{c}
\hat{v}(\xi, 1, t) \\
\hat{v}(\xi, 2, t)
\end{array}\right]=A(\xi)\left[\begin{array}{l}
\hat{v}(\xi, 1, t) \\
\hat{v}(\xi, 2, t)
\end{array}\right]
$$

where $A(\xi)$ is the $2 \times 2$ matrix,

$$
A(\xi)=\left[\begin{array}{cc}
-\gamma-\frac{2 D}{h^{2}}[1-\cos h \xi], & \gamma \\
\frac{\sigma^{2}}{h}+\frac{2 D}{h^{2}}\left[1+e^{i h \xi}\right], & \frac{-\sigma^{2}}{h}-\frac{4 D}{h^{2}}
\end{array}\right] .
$$

Let $\alpha(\xi)$ be given by

$$
\alpha(\xi)=\sqrt{\left\{\frac{\sigma^{2}}{2 h}+\frac{\gamma}{2}+\frac{D}{h^{2}}[1+\cos h \xi]\right\}^{2}+\frac{2 D \gamma}{h^{2}} i \sin h \xi},
$$

where we take the square root with positive real part. Then the eigenvalues $\lambda_{+}(\xi), \lambda_{-}(\xi)$ of $A(\xi)$ are given by,

$$
\lambda_{+}(\xi), \lambda_{-}(\xi)=-\left\{\frac{\sigma^{2}}{2 h}+\frac{\gamma}{2}+\frac{2 D}{h^{2}}+\frac{D}{h^{2}}[1-\cos h \xi]\right\} \pm \alpha(\xi) .
$$


Define $\beta(\xi)$ by

$$
\beta(\xi)=\frac{\gamma}{2}-\frac{\sigma^{2}}{2 h}-\frac{D}{h^{2}}[1+\cos h \xi] .
$$

Then the eigenvectors corresponding to $\lambda_{+}(\xi), \lambda_{-}(\xi)$ are given by $[\gamma, \beta(\xi) \pm$ $\alpha(\xi)]^{T}$. Hence the solution to the initial value problem (5.3) is given by

$$
\begin{aligned}
\hat{v}(\xi, 1, t)= & {\left[\frac{\alpha(\xi)-\beta(\xi)}{2 \alpha(\xi)} \hat{f}(\xi, 1)+\frac{\gamma}{2 \alpha(\xi)} \hat{f}(\xi, 2)\right] \exp \left[\lambda_{+}(\xi) t\right] } \\
& +\left[\frac{\alpha(\xi)+\beta(\xi)}{2 \alpha(\xi)} \hat{f}(\xi, 1)-\frac{\gamma}{2 \alpha(\xi)} \hat{f}(\xi, 2)\right] \exp \left[\lambda_{-}(\xi) t\right] \\
\hat{v}(\xi, 2, t)= & {\left[\frac{\alpha^{2}(\xi)-\beta^{2}(\xi)}{2 \gamma \alpha(\xi)} \hat{f}(\xi, 1)+\frac{\alpha(\xi)+\beta(\xi)}{2 \alpha(\xi)} \hat{f}(\xi, 2)\right] \exp \left[\lambda_{+}(\xi) t\right] } \\
& +\left[\frac{\beta^{2}(\xi)-\alpha^{2}(\xi)}{2 \gamma \alpha(\xi)} \hat{f}(\xi, 1)+\frac{\alpha(\xi)-\beta(\xi)}{2 \alpha(\xi)} \hat{f}(\xi, 2)\right] \exp \left[\lambda_{-}(\xi) t\right] .
\end{aligned}
$$

We take now $f(x, s)=e^{-\delta x}, x \geqslant 0, f(x, s)=0, x<0$, for any $\delta>0$, whence

$$
\hat{f}(\xi, s)=h /[1-\exp \{h(i \xi-\delta)\}]
$$

With $\hat{f}$ given by (5.7) we need now to estimate,

$$
v(x, 1, t)=\frac{1}{2 \pi} \int_{-\pi / h}^{\pi / h} \hat{v}(\xi, 1, t) e^{-i x \xi} d \xi
$$

We shall obtain estimates on $v(x, 1, t)$ which are independent of $\delta$ as $\delta \rightarrow 0$. We first consider

$$
\begin{aligned}
& \frac{1}{2 \pi} \int_{-\pi / h}^{\pi / h} \hat{f}(\xi, s) \exp \left[\lambda_{-}(\xi) t\right] e^{-i x \xi} d \xi \\
& \quad=\frac{1}{\pi} \operatorname{Re} \int_{0}^{\pi / h} \hat{f}(\xi, s) \exp \left[\lambda_{-}(\xi) t\right] e^{-i x \xi} d \xi
\end{aligned}
$$

Observe that

$$
\begin{aligned}
& \operatorname{Re} \hat{f}(\xi, s)=\frac{h\left(1-e^{-\delta h}\right)+h e^{-\delta h}(1-\cos h \xi)}{\left(1-e^{-\delta h}\right)^{2}+2 e^{-\delta h}(1-\cos h \xi)} \\
& \operatorname{Im} \hat{f}(\xi, s)=\frac{h e^{-\delta h} \sin h \xi}{\left(1-e^{-\delta h}\right)^{2}+2 e^{-\delta h}(1-\cos h \xi)}
\end{aligned}
$$


Hence there is a universal constant $C>0$ such that

$$
\int_{0}^{\pi / h}|\operatorname{Re} \hat{f}(\xi, s)|+|\sin h \xi \operatorname{Im} \hat{f}(\xi, s)| d \xi \leqslant C .
$$

We conclude that there is a constant $C(D, \gamma, h)$ independent of $\sigma$ such that,

$$
\begin{aligned}
& \left|\operatorname{Re} \int_{0}^{\pi / h} \hat{f}(\xi, s) \exp \left[\lambda_{-}(\xi) t\right] e^{-i x \xi} d \xi\right| \\
& \leqslant C(D, \gamma, h)[h+|x|+t] \exp [-\gamma t / 2] .
\end{aligned}
$$

Using a similar argument we see that the terms on the RHS of (5.6) involving $\exp \left[\lambda_{-}(\xi) t\right]$ are bounded by the RHS of (5.9).

In order to estimate the terms on the RHS of (5.6) which involve $\exp \left[\lambda_{+}(\xi) t\right]$ we first need to show that the real part of $\lambda_{+}(\xi)$ is strictly negative for $\xi \in[-\pi / h, \pi / h], \xi \neq 0$. We write $\lambda_{+}(\xi)=-a(\xi)+\alpha(\xi)$ where $a(\xi)>0$ and $\alpha(\xi)$ is given by (5.4). Thus,

$$
\operatorname{Re} \lambda_{+}(\xi)=\left\{-a(\xi)^{2}+[\operatorname{Re} \alpha(\xi)]^{2}\right\} /\{a(\xi)+\operatorname{Re} \alpha(\xi)\} .
$$

Observing that

$$
[\operatorname{Re} \alpha(\xi)]^{2}=\frac{1}{2}\left\{|\alpha(\xi)|^{2}+\operatorname{Re} \alpha(\xi)^{2}\right\}
$$

we conclude that,

$$
\operatorname{Re} \lambda_{+}(\xi)=\left\{-\left[a(\xi)^{2}-\operatorname{Re} \alpha(\xi)^{2}\right]-a(\xi)^{2}+|\alpha(\xi)|^{2}\right\} / 2\{a(\xi)+\operatorname{Re} \alpha(\xi)\}
$$

From (5.4), (5.5) we have that

$$
a(\xi)^{2}-\operatorname{Re} \alpha(\xi)^{2}=\left\{\frac{\sigma^{2}}{h}+\gamma+\frac{4 D}{h^{2}}\right\} \frac{2 D}{h^{2}}[1-\cos h \xi] \geqslant 0 .
$$

Hence,

$$
\begin{aligned}
\operatorname{Re} \lambda_{+}(\xi)=\{- & \left.\left(\left[a(\xi)^{2}-\operatorname{Re} \alpha(\xi)^{2}\right]+a(\xi)^{2}\right)^{2}+|\alpha(\xi)|^{4}\right\} / \\
& 2\{a(\xi)+\operatorname{Re} \alpha(\xi)\}\left\{\left[a(\xi)^{2}-\operatorname{Re} \alpha(\xi)^{2}\right]+a(\xi)^{2}+|\alpha(\xi)|^{2}\right\}
\end{aligned}
$$


The numerator of the last expression can be rewritten as

$$
\begin{aligned}
& -4 a(\xi)^{2}\left[a(\xi)^{2}-\operatorname{Re} \alpha(\xi)^{2}\right]+\left[\operatorname{Im} \alpha(\xi)^{2}\right]^{2} \\
\leqslant & -\left\{\frac{\sigma^{2}}{h}+\gamma+\frac{4 D}{h^{2}}\right\}^{3} \frac{2 D}{h^{2}}[1-\cos h \xi]+\frac{4 D^{2} \gamma^{2}}{h^{4}} \sin ^{2} h \xi \\
\leqslant & -\left\{\left[\gamma+\frac{4 D}{h^{2}}\right]^{3}-\frac{4 D \gamma^{2}}{h^{2}}\right\} \frac{2 D}{h^{2}}[1-\cos h \xi] \leqslant 0 .
\end{aligned}
$$

Hence $\operatorname{Re} \lambda_{+}(\xi)<0$ for $\xi \in[-\pi / h, \pi / h], \quad \xi \neq 0$. In fact one easily sees that there is a constant $C(D, \gamma, h)>0$ independent of $\sigma$ such that

$$
\operatorname{Re} \lambda_{+}(\xi) \leqslant-C(D, \gamma, h)[1-\cos h \xi], \quad \xi \in[-\pi / h, \pi / h] .
$$

Next we need to analytically continue $\lambda_{+}(\xi), \xi \in \mathbf{R}$, into the complex plane. To do this we use the identity,

$$
\begin{aligned}
\lambda_{+}(\xi) & =\operatorname{det}[A(\xi)] / \lambda_{-}(\xi) \\
& =\left[\left\{\frac{\sigma^{2}}{h}+\gamma+\frac{4 D}{h^{2}}\right\} \frac{2 D}{h^{2}}[1-\cos h \xi]-\frac{2 D \gamma}{h^{2}} i \sin h \xi\right] / \lambda_{-}(\xi) .
\end{aligned}
$$

It is clear from (5.11) that there are positive constants $\eta_{0}(D, \gamma, h)$, $\mathbf{C}(D, \gamma, h)$ such that $\lambda_{+}(\xi+i \eta)$ is analytic as a function of $\xi+i \eta \in \mathbf{C}$ provided $|\eta|<\eta_{0}(D, \gamma, h)$, and

$$
\left|\lambda_{+}(\xi)-\lambda_{+}(\xi+i \eta)\right| \leqslant C(D, \gamma, h)|\eta|
$$

in this region. It is also clear that for any $\eta$ satisfying $0<\eta<\eta_{0}(D, \gamma, h)$ there is the identity,

$$
\begin{aligned}
\frac{1}{2 \pi} \int_{-\pi / h}^{\pi / h} \hat{f}(\xi, s) \exp \left[\lambda_{+}(\xi) t\right] e^{-i x \xi} d \xi \\
\quad=\frac{1}{2 \pi} \int_{-\pi / h}^{\pi / h} \hat{f}(\xi+i \eta, s) \exp \left[\lambda_{+}(\xi+i \eta) t\right] \exp [-i x \xi+x \eta] d \xi
\end{aligned}
$$

For $x>0, t>0$, we define $\rho$ by

$$
\rho=2 D \gamma t /\left[\sigma^{2}+\gamma h+4 D / h\right]-x,
$$


and assume $\rho>0$. Hence $\eta=\varepsilon \rho / 2 D t$ lies in the interval $0<\eta<\eta_{0}(D, \gamma, h)$ for all $t>0$, provided $\varepsilon>0$ is chosen sufficiently small depending only on $D, \gamma, h$. Observe now the inequalities,

$$
\begin{aligned}
& |\hat{f}(\xi+i \eta, s)| \leqslant e^{(\delta+\eta) h} /(\delta+\eta), \\
& \int_{-\pi / h}^{\pi / h}|\operatorname{Re} \hat{f}(\xi+i \eta, s)|+|\sin h \xi \operatorname{Im} \hat{f}(\xi+i \eta, s)| d \xi \leqslant C,
\end{aligned}
$$

where the second inequality is just a restatement of (5.8) with $\delta>0$ replaced by $\delta+\eta$. From these inequalities, (5.10), (5.12) we get a similar inequality to (5.9). Thus there is an interval $I_{\varepsilon}=\left[-C_{1}(D, \gamma, h) \sqrt{\varepsilon}\right.$, $\left.C_{1}(D, \gamma, h) \sqrt{\varepsilon}\right]$ and constants $C_{2}(D, \gamma, h), c_{3}(D, \gamma, h)$ such that

$$
\begin{array}{r}
\left|\operatorname{Re} \int_{[-\pi / h, \pi / h]-I_{\varepsilon}} \hat{f}(\xi+i \eta, s) \exp \left[\lambda_{+}(\xi+i \eta) t\right] \exp [-i x \xi+x \eta] d \xi\right| \\
\leqslant C_{2}(D, \gamma, h)[h+|x|+t] \exp \left[-c_{3}(D, \gamma, h) \varepsilon t\right] .
\end{array}
$$

Finally we need to estimate the integral on the interval $I_{\varepsilon}$ where we may assume $h \xi$ is small. To do this we write

$$
\begin{aligned}
\exp \left[\lambda_{+}(\xi+i \eta) t\right]= & \exp \left[\operatorname{Re} \lambda_{+}(\xi+i \eta) t+i \operatorname{Im} \lambda_{+}(\xi) t\right] \\
& \times\left\{1+\left(\exp \left[i \operatorname{Im}\left\{\lambda_{+}(\xi+i \eta)-\lambda_{+}(\xi)\right\} t\right]-1\right)\right\} .
\end{aligned}
$$

Observe that there is a constant $C_{4}(D, \gamma, h)$, such that

$$
\left|\frac{\lambda_{-}(0)}{\lambda_{-}(\xi+i \eta)}-1+\frac{2 D \gamma h i(\xi+i \eta)}{\left(\sigma^{2}+\gamma h+4 D / h\right)^{2}}\right| \leqslant C_{4}(D, \gamma, h)|\xi+i \eta|^{2} .
$$

Hence from (5.11) there is the inequality,

$$
\begin{aligned}
& \left|\lambda_{+}(\xi+i \eta)-\frac{2 D \gamma i(\xi+i \eta)}{\sigma^{2}+\gamma h+4 D / h}+D\left\{1-\frac{4 D \gamma^{2} h}{\left(\sigma^{2}+\gamma h+4 D / h\right)^{3}}\right\}(\xi+i \eta)^{2}\right| \\
& \leqslant C_{5}(D, \gamma, h)|\xi+i \eta|^{3} \text {. }
\end{aligned}
$$

Thus for $\varepsilon$ sufficiently small, depending only on $D, \gamma, h$ there is the inequality,

$$
\operatorname{Re} \lambda_{+}(\xi+i \eta) \leqslant-\frac{D \xi^{2}}{2}+2 D \eta^{2}-\frac{2 D \gamma \eta}{\sigma^{2}+\gamma h+4 D / h} .
$$


From the previous inequality one has that

$$
\operatorname{Re} \lambda_{+}(\xi+i \eta) t+x \eta \leqslant-D \xi^{2} t / 2-\varepsilon(1-\varepsilon) \rho^{2} / 2 D t .
$$

The result follows now from (5.13), (5.14), (5.15), (5.16).

Corollary 5.1. Let $c_{h}(\sigma)$ be the wave speed for the discretized equation (5.1). Then there is the inequality,

$$
c_{h}(\sigma) \geqslant 2 D \gamma /\left[\sigma^{2}+\gamma h+4 D / h\right] .
$$

Proof. We proceed as in Lemma 5.4 and use Lemma 5.5 and Lemma 5.6. Thus from (5.2) one has for $0<x<2 D \gamma t /\left[\sigma^{2}+\gamma h+4 D / h\right]$ the inequality,

$$
\langle 1-u(x, t)\rangle \leqslant \frac{C(D, \gamma, h)}{\sqrt{t}} \exp \left[\frac{-\rho^{2}}{C(D, \gamma, h) t}\right] .
$$

Now we follow the argument of Lemma 5.4.

Remark 1. The lower bound on $c_{h}(\sigma)$ in Corollary 5.1 is consistent with the conjecture $c_{h}(\sigma) \sim 2 D \gamma / \sigma^{2}$ as $\sigma \rightarrow \infty$ stated in ref. 5 .

The lower bound on $c_{h}(\sigma)$ in Corollary 5.1 goes to 0 as $h \rightarrow 0$. In order to get a positive lower bound on $c(\sigma)$ we need an estimate which remains positive as $h \rightarrow 0$. To do this we define a Markov chain similar to but somewhat more complicated than the Markov chain studied in Lemma 5.6. The state space for the chain is $h \mathbf{Z} \cup(h \mathbf{Z} \times h \mathbf{Z})$. The transitions in the chain occur at exponential rates as follows:

$$
\begin{aligned}
x & \rightarrow x+h \text { at rate } D / h^{2}, x \in h \mathbf{Z}, \\
x & \rightarrow x-h \text { at rate } D / h^{2}, x \in h \mathbf{Z}, \\
x & \rightarrow(x, x) \in h \mathbf{Z} \times h \mathbf{Z} \text { at rate } \gamma, x \in h \mathbf{Z}, \\
(x, y) & \rightarrow(x+h, y) \text { at rate } D / h^{2}, x, y \in h \mathbf{Z}, \\
(x, y) & \rightarrow(x-h, y) \text { at rate } D / h^{2}, x, y \in h \mathbf{Z}, \\
(x, y) & \rightarrow(x, y+h) \text { at rate } D / h^{2}, x, y \in h \mathbf{Z}, \\
(x, y) & \rightarrow(x, y-h) \text { at rate } D / h^{2}, x, y \in h \mathbf{Z}, \\
(x, x) & \rightarrow x \in h \mathbf{Z} \text { at rate } \sigma^{2} / h, x \in h \mathbf{Z}, \\
(x, y) & \rightarrow \min [x, y] \in h \mathbf{Z} \text { at rate } \lambda_{\sigma}, x, y \in h \mathbf{Z} .
\end{aligned}
$$


The final rate $\lambda_{\sigma}>0$ has yet to be determined. Any positive value of $\lambda_{\sigma}$ yields a positive lower bound on $c(\sigma)$. We shall see later how to choose $\lambda_{\sigma}$ to obtain an optimal lower bound on $c(\sigma)$ for large $\sigma$.

Lemma 5.7. Let $X_{h}(t)$ be the Markov chain on $h \mathbf{Z} \cup(h \mathbf{Z} \times h \mathbf{Z})$ defined by the transition rates (5.17). Then there is the inequality,

$$
\begin{aligned}
& P\left[X_{h}(t) \text { has at least one coordinate which is not positive } \mid X_{h}(0)=x \in h \mathbf{Z}\right] \\
& \quad \leqslant P\left[N_{j}(t)>0 \text { for some } \quad j \leqslant 0 \mid N_{i}(0)=0, i \neq x / h, N_{x / h}(0)=1\right] .
\end{aligned}
$$

Proof. We proceed as in Lemma 5.5. Thus for the many particle system all but 1 or 2 particles are black and the remainder red. The transition $(x, y) \rightarrow \min [x, y]$ corresponds to the right most of 2 red particles turning black.

We wish now to estimate the probability that the Markov chain $X_{h}(t)$ started at $x \in h \mathbf{Z}$ has all co-ordinates positive. Since the chain is more complicated than the one considered in Lemma 5.6 we shall proceed differently. We define random variables $\tau_{h}, Y_{h}(t), t<\tau_{h}$, as follows: Consider $X_{h}(t)$ with $\lambda_{\sigma}=0$ started at $(0,0) \in h \mathbf{Z} \times h \mathbf{Z}$. Then $\tau_{h}=\inf \{t>0$ : $\left.X_{h}(t) \in h \mathbf{Z}\right\}$. For $0<t \leqslant \tau_{h}$ the variable $Y_{h}(t)$ is the minimum of the 2 coordinates of $X_{h}(t) \in h \mathbf{Z} \times h \mathbf{Z}$. We define functions $a_{h}, b_{h}$ as

$$
\begin{aligned}
& a_{h}(\theta, t)=E\left[\exp \left\{\theta Y_{h}(t)\right\} ; t<\tau_{h}\right], \theta, t>0, \\
& b_{h}(\theta, t)=E\left[\exp \left\{\theta Y_{h}(t)\right\} \mid \tau_{h}=t\right], \theta, t>0 .
\end{aligned}
$$

With $\lambda_{\sigma}$ as in (5.17) let $T_{1}, T_{2}, \ldots$, be i.i.d. exponential variables with parameter $\lambda_{\sigma}$. Let $\tau_{h, 1}, \tau_{h, 2}, \ldots$, be i.i.d variables which have the same distribution as the variable $\tau_{h}$ defined above.

Lemma 5.8. Let $T_{\gamma}$ be an exponential variable with parameter $\gamma$. Then for $\theta>0$ there is the inequality,

$$
\begin{aligned}
& P\left(\text { all coordinates of } X_{h}(t) \text { are positive } \mid X_{h}(0)=x \in h \mathbf{Z}\right) \\
& \leqslant e^{\theta x} \sum_{m, k=0}^{\infty}\left(\begin{array}{c}
m+k \\
m
\end{array}\right) E\left\{\prod_{r=1}^{m} a_{h}\left(\theta, T_{r}\right) \sup _{0<s<T_{m+1}} a_{h}(\theta, s)\right. \\
& \times \prod_{r=1}^{k} b_{h}\left(\theta, \tau_{h, r}\right) \exp \left[-\lambda_{\sigma}\left\{\tau_{h, 1}+\cdots+\tau_{h, k}\right\}\right] \exp \left[\frac{2 D}{h^{2}}\{\cosh (\theta h)-1\}\left\{t-T_{h, m, k}\right\}\right] \\
& \left.\times \int_{\left(t-T_{h, m, k}-T_{\gamma}-T_{m+1}\right)^{+}}^{\left(t-T_{h, m, k}\right)^{+}} \frac{(\gamma s)^{m+k-1}}{(m+k-1) !} e^{-\gamma s} \gamma d s\right\},
\end{aligned}
$$


where $T_{h, m, k}$ is the random variable,

$$
T_{h, m, k}=T_{1}+\cdots+T_{m}+\tau_{h, 1}+\cdots+\tau_{h, k}
$$

The term in the summation corresponding to $m=k=0$ is given by

$$
\exp \left[\frac{2 D}{h^{2}}\{\cosh (\theta h)-1\} t\right] E\left[\sup _{0<s<T_{1} \wedge t} a_{h}(\theta, s) ; T_{\gamma}+T_{1}>t\right]
$$

Proof. We need to show that the RHS of the inequality is an upper bound for

$$
E\left[\exp \left\{\theta \bar{X}_{h}(t)\right\} \mid X_{h}(0)=x \in h \mathbf{Z}\right]
$$

where $\bar{X}_{h}(t)$ is the minimum of the co-ordinates of $X_{h}(t)$. We consider first the situation where $X_{h}(s) \in h \mathbf{Z}, 0 \leqslant s \leqslant t$. It is evident that

$$
\begin{aligned}
E\left[\exp \left\{\theta \bar{X}_{h}(t)\right\} \mid X_{h}(0)\right. & \left.=0, X_{h}(s) \in h \mathbf{Z}, 0 \leqslant s \leqslant t\right] \\
& =\exp \left[\frac{2 D}{h^{2}}\{\cosh (\theta h)-1\} t\right], \\
P\left(X_{h}(s) \in h \mathbf{Z}, 0 \leqslant s \leqslant t\right) & =P\left(T_{\gamma}>t\right) .
\end{aligned}
$$

Next we consider the case where a particle is created at time $T_{\gamma}<t$ and $X_{h}(s) \in h \mathbf{Z} \times h \mathbf{Z}$ for $T_{\gamma}<s \leqslant t$. This corresponds to the inequalities,

$$
T_{\gamma}<t<T_{\gamma}+\min \left[T_{1}, \tau_{h, 1}\right]
$$

Suppose now $T_{\gamma}, T_{1}$ are fixed and we take the expectation on the event (5.21),

$$
\begin{aligned}
E & {\left[\exp \left\{\theta \bar{X}_{h}(t)\right\} ; \text { no particle deletion before time } t \mid X_{h}(0)=0, T_{\gamma}, T_{1}\right] } \\
& =\exp \left[\frac{2 D}{h^{2}}\{\cosh (\theta h)-1\} T_{\gamma}\right] a_{h}\left(\theta, t-T_{\gamma}\right) \\
& \leqslant \exp \left[\frac{2 D}{h^{2}}\{\cosh (\theta h)-1\} t\right] \sup _{0<s<T_{1} \wedge t} a_{h}(\theta, s)
\end{aligned}
$$

We conclude then from (5.20), (5.22) that 
$\mathrm{E}\left[\exp \left\{\theta \bar{X}_{h}(t)\right\}\right.$; at most 1 particle creation and no deletion by time $\left.t \mid X_{h}(0)=0\right]$

$$
\leqslant \exp \left[\frac{2 D}{h^{2}}\{\cosh (\theta h)-1\} t\right] E\left[\sup _{0<s<T_{1} \wedge t} a_{h}(\theta, s) ; T_{\gamma}+T_{1}>t\right] .
$$

The RHS of the last inequality is the same as (5.19).

We consider now the situation where there are $m+k \geqslant 1$ particle deletions before time $t, m$ of these occurring by virtue of the final transition in (5.17) and $k$ of them by virtue of the penultimate transition of (5.17). Note that there are

$$
\left(\begin{array}{c}
m+k \\
m
\end{array}\right)
$$

ways of ordering the particle deletions. Let $T_{1}, \ldots, T_{m}$ be the times from creation to deletion for particles which are deleted by virtue of the final transition in (5.17). Then $T_{1}, \ldots, T_{m}$ are independent exponential variables with parameter $\lambda_{\sigma}$. Similarly let $\tau_{h, 1}, \ldots, \tau_{h, k}$ be the times from creation to deletion for particles deleted by virtue of the penultimate transition of (5.17). The variables $\tau_{h, 1}, \ldots, \tau_{h, k}$ are i.i.d. with distribution the same as $\tau_{h}$. Then we have

$E\left[\exp \left\{\theta \bar{X}_{h}(t)\right\} ; m+k\right.$ particle creations and $m+k$ particle deletions before time $t, m$ of the deletions being by virtue of the final transition of $\left.(5.17) \mid X_{h}(0)=0, T_{1}, \ldots, T_{m}, \tau_{h, 1}, \ldots, \tau_{h, k}\right]$

$$
\begin{aligned}
& =\left(\begin{array}{c}
m+k \\
m
\end{array}\right) \prod_{r=1}^{m} a_{h}\left(\theta, T_{r}\right) \prod_{r=1}^{k} b_{h}\left(\theta, \tau_{h, r}\right) \exp \left[-\lambda_{\sigma}\left\{\tau_{h, 1}+\cdots+\tau_{h, k}\right\}\right] \\
& \times \exp \left[\frac{2 D}{h^{2}}\{\cosh (\theta h)-1\}\left\{t-T_{h, m, k}\right\}\right],
\end{aligned}
$$

where $T_{h, m, k}<t$ is given by (5.18). For $n>0$ let $T_{n, \gamma}$ be a Gamma variable with parameters $(n, \gamma)$. The event in (5.23) corresponds to the inequality,

$$
T_{m+k, \gamma}+T_{h, m, k}<t<T_{\gamma}+T_{m+k, \gamma}+T_{h, m, k}
$$


The situation where there are $m+k+1$ particle creations and $m+k$ particle deletions before time $t$ corresponds to the inequality,

$$
T_{\gamma}+T_{m+k, \gamma}+T_{h, m, k}<t<\min \left[T_{m+1}, \tau_{h, k+1}\right]+T_{\gamma}+T_{m+k, \gamma}+T_{h, m, k}
$$

We have then the identity,

$$
\begin{aligned}
& E\left[\exp \left\{\theta \bar{X}_{h}(t)\right\} ; m+k+1 \text { particle creations and } m+k\right. \text { particle de } \\
& \text { before time } t, m \text { of the deletions being by virtue of the final transt } \\
& \text { of } \left.(5.17) \mid X_{h}(0)=0, T_{1}, \ldots, T_{m+1}, \tau_{h, 1}, \ldots, \tau_{h, k}, T_{\gamma}, T_{m+k, \gamma}\right] \\
& =\left(\begin{array}{c}
m+k \\
m
\end{array}\right) \prod_{r=1}^{m} a_{h}\left(\theta, T_{r}\right) a_{h}\left(\theta, t-T_{\gamma}-T_{m+k, \gamma}-T_{h, m, k}\right) \\
& \quad \times \prod_{r=1}^{k} b_{h}\left(\theta, \tau_{h, r}\right) \exp \left[-\lambda_{\sigma}\left\{\tau_{h, 1}+\cdots+\tau_{h, k}\right\}\right] \\
& \quad \times \exp \left[\frac{2 D}{h^{2}}\{\cosh (\theta h)-1\}\left\{T_{\gamma}+T_{m+k, \gamma}\right\}\right]
\end{aligned}
$$

Observe now that in view of (5.25) one has

$$
a_{h}\left(\theta, t-T_{\gamma}-T_{m+k, \gamma}-T_{h, m, k}\right) \leqslant \sup _{0 \leqslant s<T_{m+1}} a_{h}(\theta, s) .
$$

We also have from (5.25) that $T_{\gamma}+T_{m+k, \gamma}<t-T_{h, m, k}$. Observe also that the events (5.24), (5.25) are disjoint and that the union of these events is contained in the event,

$$
t-T_{h, m, k}-T_{\gamma}-T_{m+1}<T_{m+k, \gamma}<t-T_{h, m, k} .
$$

We conclude then that

$E\left[\exp \left\{\theta \bar{X}_{h}(t)\right\} ; m+k\right.$ or $m+k+1$ particle creations and $m+k$ deletions before time $t, m$ of the deletions being by virtue of the final transition of 


$$
\begin{aligned}
X_{h}(0)= & \left.0, T_{1}, \ldots, T_{m+1}, \tau_{h, 1}, \ldots, \tau_{h, k}, T_{\gamma}\right] \\
\leqslant & \left(\begin{array}{c}
m+k \\
m
\end{array}\right) \prod_{r=1}^{m} a_{h}\left(\theta, T_{r}\right) \sup _{0 \leqslant s<T_{m+1}} a_{h}(\theta, s) \\
& \times \prod_{r=1}^{k} b_{h}\left(\theta, \tau_{h, r}\right) \exp \left[-\lambda_{\sigma}\left\{\tau_{h, 1}+\cdots+\tau_{h, k}\right\}\right] \\
& \times \exp \left[\frac{2 D}{h^{2}}\{\cosh (\theta h)-1\}\left\{t-T_{h, m, k}\right\}\right] \\
& \times \int_{\left(t-T_{h, m, k}-T_{\gamma}-T_{m+1}\right)^{+}}^{\left(t-T_{h, m, k}\right)^{+}} \frac{(\gamma s)^{m+k-1}}{(m+k-1) !} e^{-\gamma s} \gamma d s .
\end{aligned}
$$

The result follows from this last inequality on taking the expectation and summing w.r.t. $m, k$.

We need to estimate the RHS of the inequality in the statement of Lemma 5.8 in such a way that our estimates are uniform as $h \rightarrow 0$. We begin with the function $a_{h}(\theta, t)$. To do this we define a Markov chain with state space $\left\{\left(y_{1}, y_{2}\right) \in h \mathbf{Z} \times h \mathbf{Z}: y_{1} \geqslant y_{2}\right\}$. The transitions in the chain occur at exponential rates as follows:

$$
\begin{aligned}
& \left(y_{1}, y_{2}\right) \rightarrow\left(y_{1}+h, y_{2}\right) \quad \text { at rate } D / h^{2}, y_{1}>y_{2}, \\
& \left(y_{1}, y_{2}\right) \rightarrow\left(y_{1}-h, y_{2}\right) \quad \text { at rate } D / h^{2}, y_{1}>y_{2}, \\
& \left(y_{1}, y_{2}\right) \rightarrow\left(y_{1}, y_{2}+h\right) \quad \text { at rate } D / h^{2}, y_{1}>y_{2}, \\
& \left(y_{1}, y_{2}\right) \rightarrow\left(y_{1}, y_{2}-h\right) \quad \text { at rate } D / h^{2}, y_{1}>y_{2}, \\
& \left(y_{1}, y_{2}\right) \rightarrow\left(y_{1}+h, y_{2}\right) \quad \text { at rate } 2 D / h^{2}, y_{1}=y_{2}, \\
& \left(y_{1}, y_{2}\right) \rightarrow\left(y_{1}, y_{2}-h\right) \quad \text { at rate } 2 D / h^{2}, y_{1}=y_{2}, \\
& \left(y_{1}, y_{2}\right) \rightarrow \text { killed at rate } \sigma^{2} / h, y_{1}=y_{2},
\end{aligned}
$$

Let $\tau_{h}$ be the killing time for the Markov chain defined by (5.27). Then if the position of the walk at time $t$ is $\left(Y_{h, 1}(t), Y_{h, 2}(t)\right) \in h \mathbf{Z} \times h \mathbf{Z}$, one has the identity,

$$
a_{h}(\theta, t)=E\left[\exp \left\{-\theta Y_{h, 1}(t)\right\} ; t<\tau_{h} \mid Y_{h, 1}(0)=Y_{h, 2}(0)=0\right] .
$$

Let $f$ be a real valued function on the state space of the chain (5.27) and consider

$$
u_{h}\left(y_{1}, y_{2}, t\right)=E\left[f\left(Y_{h, 1}(t), Y_{h, 2}(t)\right) ; t<\tau_{h} \mid Y_{h, 1}(0)=y_{1}, Y_{h, 2}(0)=y_{2}\right] .
$$


Then $u_{h}$ satisfies the partial difference equation,

$$
\begin{aligned}
\frac{\partial u_{h}}{\partial t}\left(y_{1}, y_{2}, t\right)=\frac{D}{h^{2}}[ & u_{h}\left(y_{1}+h, y_{2}, t\right)+u_{h}\left(y_{1}-h, y_{2}, t\right)+u_{h}\left(y_{1}, y_{2}+h, t\right) \\
& \left.+u_{h}\left(y_{1}, y_{2}-h, t\right)-4 u_{h}\left(y_{1}, y_{2}, t\right)\right], \quad y_{1}>y_{2}, \quad(5.28) \\
\frac{\partial u_{h}}{\partial t}\left(y_{1}, y_{2}, t\right)= & \frac{2 D}{h^{2}}\left[u_{h}\left(y_{1}+h, y_{2}, t\right)+u_{h}\left(y_{1}, y_{2}-h, t\right)-2 u_{h}\left(y_{1}, y_{2}, t\right)\right] \\
& -\frac{\sigma^{2}}{h} u_{h}\left(y_{1}, y_{2}, t\right), \quad y_{1}=y_{2}
\end{aligned}
$$

for $t>0$ with initial condition,

$$
u_{h}\left(y_{1}, y_{2}, 0\right)=f\left(y_{1}, y_{2}\right), \quad y_{1} \geqslant y_{2}
$$

Next we define a Markov chain with state space $\{z \in h \mathbf{Z}: z \geqslant 0\}$. The transitions in the chain occur at exponential rates as follows:

$$
\begin{aligned}
& z \rightarrow z+h \text { at rate } 2 D / h^{2}, \quad z>0 \\
& z \rightarrow z-h \text { at rate } 2 D / h^{2}, \quad z>0 \\
& 0 \rightarrow h \text { at rate } 4 D / h^{2}, \\
& 0 \rightarrow \text { killed at rate } \sigma^{2} / h .
\end{aligned}
$$

Let $Z_{h}(t)$ be the position of the walk at time $t$ determined by (5.30). For a real valued function $g$ on the state space of the chain (5.30) we put,

$$
v_{h}(z, t)=E\left[g\left(Z_{h}(t)\right) ; t<\tau_{h} \mid Z_{h}(0)=z\right]
$$

where $\tau_{h}$ is the killing time for the chain. Then $v_{h}$ satisfies the partial difference equation,

$$
\begin{aligned}
& \frac{\partial v_{h}}{\partial t}(z, t)=\frac{2 D}{h^{2}}\left[v_{h}(z+h, t)+v_{h}(z-h, t)-2 v_{h}(z, t)\right], \quad z>0 \\
& \frac{\partial v_{h}}{\partial t}(0, t)=\frac{4 D}{h^{2}}\left[v_{h}(h, t)-v_{h}(0, t)\right]-\frac{\sigma^{2}}{h} v_{h}(0, t)
\end{aligned}
$$


for $t>0$ with initial condition,

$$
v_{h}(z, 0)=g(z), \quad z \geqslant 0
$$

Observe that there is a relation between the chains (5.27), (5.30). Thus if $v_{h}(z, t)$ is a solution of (5.32) with initial condition (5.33) then $u_{h}\left(y_{1}, y_{2}, t\right)$ $=v_{h}\left(y_{1}-y_{2}, t\right)$ is a solution of (5.28) with initial condition (5.29) given by $f\left(y_{1}, y_{2}\right)=g\left(y_{1}-y_{2}\right)$. In terms of expectations we have the identity,

$$
\begin{aligned}
& E\left[g\left(Y_{h, 1}(t)-Y_{h, 2}(t)\right) ; t<\tau_{h} \mid Y_{h, 1}(0)=y_{1}, \quad Y_{h, 2}(0)=y_{2}\right] \\
& \quad=E\left[g\left(Z_{h}(t)\right) ; t<\tau_{h} \mid Z_{h}(0)=y_{1}-y_{2}\right] .
\end{aligned}
$$

We can extend the identity (5.34) by an inequality when the function $f\left(y_{1}, y_{2}\right)$ is no longer just a function of $y_{1}-y_{2}$.

Lemma 5.9. Let $u_{h}$ be the solution of (5.28) with initial condition $f$ given by

$$
f\left(y_{1}, y_{2}\right)=e^{-\theta\left(y_{1}+y_{2}\right) / 2} g\left(y_{1}-y_{2}\right), \quad y_{1} \geqslant y_{2} \text {. }
$$

Let $v_{h, \theta}$ be the solution of (5.32) with the diffusion constant $D$ in the equation replaced by $D \cosh (\theta h / 2)$ and with the initial condition (5.33). Then there is the identity,

$$
\begin{aligned}
u_{h}\left(y_{1}, y_{2}, t\right)=\exp & {\left[-\theta\left(y_{1}+y_{2}\right) / 2\right.} \\
+ & \left.\frac{4 D}{h^{2}}\left\{\cosh \left(\frac{\theta h}{2}\right)-1\right\} t\right] v_{h, \theta}\left(y_{1}-y_{2}, t\right), \quad y_{1} \geqslant y_{2}, t>0 .
\end{aligned}
$$

Proof. If we take $u_{h}$ to be the RHS of (5.36) then one can easily see that it has initial condition (5.35). One can also see that for this function the LHS of (5.28) is larger than the RHS. The inequality (5.36) follows now by the maximum principle.

Lemma 5.9 will enable us to estimate the function $a_{h}(\theta, t)$. Next we turn to the problem of estimating the function $b_{h}(\theta, t)$. To do this we consider an elliptic problem. Let $f$ be a real valued function on the state space of the chain (5.27) and for any $\lambda>0$ consider

$$
u_{h}\left(y_{1}, y_{2}\right)=E\left[f\left(Y_{h, 1}\left(\tau_{h}\right), Y_{h, 2}\left(\tau_{h}\right)\right) e^{-\lambda \tau_{h}} \mid Y_{h, 1}(0)=y_{1}, Y_{h, 2}(0)=y_{2}\right] .
$$


Then $u_{h}$ satisfies the partial difference equation,

$$
\begin{gathered}
\left(\frac{4 D}{h^{2}}+\lambda\right) u_{h}\left(y_{1}, y_{2}\right)=\frac{D}{h^{2}}\left[u_{h}\left(y_{1}+h, y_{2}\right)+u_{h}\left(y_{1}-h, y_{2}\right)\right. \\
\left.\quad+u_{h}\left(y_{1}, y_{2}+h\right)+u_{h}\left(y_{1}, y_{2}-h\right)\right], \quad y_{1}>y_{2} \\
\left(\frac{4 D}{h^{2}}+\frac{\sigma^{2}}{h}+\lambda\right) u_{h}\left(y_{1}, y_{2}\right)= \\
\quad \frac{2 D}{h^{2}}\left[u_{h}\left(y_{1}+h, y_{2}\right)+u_{h}\left(y_{1}, y_{2}-h\right)\right] \\
+\frac{\sigma^{2}}{h} f\left(y_{1}, y_{2}\right), \quad y_{1}=y_{2} .
\end{gathered}
$$

If we take $f \equiv 1$ in (5.37) then we obtain an identity analogous to (5.34). That is one has $u_{h}\left(y_{1}, y_{2}\right)=v_{h}\left(y_{1}-y_{2}\right)$ where $v_{h}(z), z \geqslant 0$, satisfies the partial difference equation,

$$
\begin{aligned}
\left(\frac{4 D}{h^{2}}+\lambda\right) v_{h}(z) & =\frac{2 D}{h^{2}}\left[v_{h}(z+h)+v_{h}(z-h)\right], \quad z>0, \\
\left(\frac{4 D}{h^{2}}+\frac{\sigma^{2}}{h}+\lambda\right) v_{h}(0) & =\frac{2 D}{h^{2}} v_{h}(h)+\frac{\sigma^{2}}{h}
\end{aligned}
$$

Lemma 5.10. Let $u_{h}$ be the solution of (5.38) with the function $f$ given by

$$
f\left(y_{1}, y_{2}\right)=e^{-\theta\left(y_{1}+y_{2}\right) / 2}, \quad y_{1} \geqslant y_{2},
$$

and $\lambda$ satisfying the inequality

$$
\lambda>\frac{4 D}{h^{2}}\left[\cosh \left(\frac{\theta h}{2}\right)-1\right] .
$$

Let $v_{h, \theta}$ be the solution of (5.39) with the diffusion constant $D$ in the equation replaced by $D \cosh (\theta h / 2)$ and $\lambda$ in the equation replaced by $\lambda-$ $\frac{4 D}{h^{2}}[\cosh (\theta h / 2)-1]$. Then there is the identity,

$$
u_{h}\left(y_{1}, y_{2}\right)=e^{-\theta\left(y_{1}+y_{2}\right) / 2} v_{h, \theta}\left(y_{1}-y_{2}\right), \quad y_{1} \geqslant y_{2}
$$


Proof. Substitute the ansatz for $u_{h}$ into (5.38) and use (5.39) to verify that (5.38) holds.

We can use Lemmas 5.9 and 5.10 to bound the terms on the RHS of the inequality in Lemma 5.8.

Lemma 5.11. Let $e^{\theta x} \sum_{m, k=0}^{\infty} A_{m, k}$ be the RHS of the inequality in the statement of Lemma 5.8. Let $Z_{h}(t)$ be the walk defined by (5.30) with the diffusion constant $D$ replaced by $D \cosh (\theta h / 2)$. Let $\delta_{h, \sigma}(\theta)$ be defined by,

$$
\delta_{h, \sigma}(\theta)=\int_{0}^{\infty} E\left[1-\exp \left\{-\theta Z_{h}(t) / 2\right\} ; t<\tau_{h} \mid Z_{h}(0)=0\right] e^{-\lambda_{\sigma} t} \lambda_{\sigma} d t .
$$

Then for $N=0,1,2, \ldots$, there is the inequality,

$$
\begin{aligned}
\sum_{m+k=N} A_{m, k} \leqslant & \exp \left[\frac{4 D}{h^{2}}\{\cosh (\theta h)-1\} t\right]\left\{1-\delta_{h, \sigma}(\theta)\right\}^{N} \\
& \times \int_{0}^{t} \frac{(\gamma s)^{N-1}}{(N-1) !} e^{-\gamma s} \gamma d s .
\end{aligned}
$$

Proof. For $N=0$ the integral in (5.41) is not part of the inequality. It follows now from (5.19), (5.36) and the inequality,

$$
\frac{4 D}{h^{2}}\left\{\cosh \left(\frac{\theta h}{2}\right)-1\right\} \leqslant \frac{2 D}{h^{2}}\{\cosh (\theta h)-1\},
$$

that (5.41) holds for $N=0$. Consider now $N \geqslant 1$ and $m, k$ fixed with $m+$ $k=N$. Then there is the inequality,

$$
\begin{aligned}
A_{m, k} \leqslant & \left(\begin{array}{c}
m+k \\
m
\end{array}\right) \exp \left[\frac{2 D}{h^{2}}\{\cosh (\theta h)-1\} t\right] \\
& \times \prod_{r=1}^{m} E\left[a_{h}\left(\theta, T_{r}\right) \exp \left[-\frac{2 D}{h^{2}}\{\cosh (\theta h)-1\} T_{r}\right]\right] \\
\times E & {\left[\sup _{0<s<T_{m+1} \wedge t} a_{h}(\theta, s)\right] \prod_{r=1}^{k} E\left[\operatorname { e x p } \left\{-\frac{\theta}{2}\left[Y_{h, 1}\left(\tau_{h, r}\right)+Y_{h, 2}\left(\tau_{h, r}\right)\right]\right.\right.} \\
& \left.\left.-\left[\lambda_{\sigma}+\frac{2 D}{h^{2}}\{\cosh (\theta h)-1\}\right] \tau_{h, r}\right\} \mid Y_{h, 1}(0)=Y_{h, 2}(0)=0\right] \\
& \times \int_{0}^{t} \frac{(\gamma s)^{N-1}}{(N-1) !} e^{-\gamma s} \gamma d s .
\end{aligned}
$$


By Lemma 5.9 and (5.42) there is the inequality,

$$
\begin{aligned}
& E\left[a_{h}\left(\theta, T_{r}\right) \exp \left[-\frac{2 D}{h^{2}}\{\cosh (\theta h)-1\} T_{r}\right]\right] \\
& \leqslant \int_{0}^{\infty} E\left[\exp \left\{-\theta Z_{h}(t) / 2\right\} ; t<\tau_{h} \mid Z_{h}(0)=0\right] e^{-\lambda_{\sigma} t} \lambda_{\sigma} d t \\
& \quad=\int_{0}^{\infty} P\left(t<\tau_{h} \mid Z_{h}(0)=0\right) e^{-\lambda_{\sigma} t} \lambda_{\sigma} d t-\delta_{h, \sigma}(\theta),
\end{aligned}
$$

where $Z_{h}$ is the random walk defined by (5.30) but with the diffusion constant $D$ replaced by $D \cosh (\theta h / 2)$. From Lemma 5.10 and (5.42) there is the inequality,

$$
\begin{gathered}
E\left[\exp \left\{-\frac{\theta}{2}\left[Y_{h, 1}\left(\tau_{h, r}\right)+Y_{h, 2}\left(\tau_{h, r}\right)\right]-\left[\lambda_{\sigma}+\frac{2 D}{h^{2}}\{\cosh (\theta h)-1\}\right] \tau_{h, r}\right\}\right. \\
\left.\mid Y_{h, 1}(0)=Y_{h, 2}(0)=0\right] \leqslant E\left[e^{-\lambda_{\sigma} \tau_{h}} \mid Z_{h}(0)=0\right],
\end{gathered}
$$

where again $Z_{h}$ is the random walk defined by (5.30) with $D$ replaced by $D \cosh (\theta h / 2)$. Observe now that

$$
\int_{0}^{\infty} P\left(t<\tau_{h} \mid Z_{h}(0)=0\right) e^{-\lambda_{\sigma} t} \lambda_{\sigma} d t+E\left[e^{-\lambda_{\sigma} \tau_{h}} \mid Z_{h}(0)=0\right]=1 .
$$

The inequality (5.41) follows now from the previous inequalities.

Lemma 5.11 enables us to find a bound on the probability in the statement of Lemma 5.8. This bound grows however exponentially in $t$ as $t \rightarrow \infty$. By a slight modification of the argument of Lemma 5.11 we can obtain a bound which decays exponentially in $t$ as $t \rightarrow \infty$.

Lemma 5.12. Let $\alpha$ satisfy the inequalities $0<\alpha<1, \alpha \gamma<\lambda_{\sigma}$. Define $\delta_{h, \sigma, \alpha}(\theta)$ by (5.40) but with $\lambda_{\sigma}$ replaced by $\lambda_{\sigma}-\alpha \gamma>0$. Suppose $\alpha$ also satisfies the inequality,

$$
\alpha\left[1+\gamma / \lambda_{\sigma}\right]<\delta_{h, \sigma, \alpha}(\theta) .
$$

Then there is the inequality,

$$
\begin{aligned}
& P\left(\text { all coordinates of } X_{h}(t) \quad \text { are positive } \mid X_{h}(0)=x \in h \mathbf{Z}\right) \\
& \quad \leqslant \frac{\alpha^{-1} \lambda_{\sigma}(1-\alpha)}{\alpha^{2} \gamma+\lambda_{\sigma} \delta_{h, \sigma, \alpha}(\theta)-\alpha\left(\gamma+\lambda_{\sigma}\right)} \exp \left[\theta x+\frac{4 D}{h^{2}}\{\cosh (\theta h)-1\} t-\alpha \gamma t\right]
\end{aligned}
$$


Proof. We use the notation of Lemma 5.11. From the inequality,

$$
(\gamma s)^{N-1} /(N-1) ! \leqslant(1-\alpha)^{-N+1} \exp [(1-\alpha) \gamma s]
$$

we have that for $m+k=N \geqslant 1$,

$$
\begin{aligned}
& E\left[\int_{\left(t-T_{h, m, k}-T_{\gamma}-T_{m+1}\right)^{+}}^{\left(t-T_{h, m, k}\right)^{+}} \frac{(\gamma s)^{N-1}}{(N-1) !} e^{-\gamma s} \gamma d s \mid T_{h, m, k}\right] \\
& \leqslant \frac{1}{\alpha(1-\alpha)^{N-1}} \exp \left[-\alpha \gamma t+\alpha \gamma T_{h, m, k}\right] E\left[\exp \left\{\alpha \gamma\left(T_{\gamma}+T_{m+1}\right)\right\}\right] .
\end{aligned}
$$

Since $T_{\gamma}$ and $T_{m+1}$ are exponential variables with parameters $\gamma, \lambda_{\sigma}$ respectively one has that

$$
E\left[\exp \left\{\alpha \gamma\left(T_{\gamma}+T_{m+1}\right)\right\}\right]=\frac{\lambda_{\sigma}}{(1-\alpha)\left(\lambda_{\sigma}-\alpha \gamma\right)},
$$

where we have used the assumptions $\alpha<1, \alpha \gamma<\lambda_{\sigma}$. We conclude that the expectation on the LHS of (5.45) is bounded by

$$
\frac{1}{\alpha(1-\alpha)^{N}} \frac{\lambda_{\sigma}}{\lambda_{\sigma}-\alpha \gamma} \exp \left[\alpha \gamma T_{h, m, k}-\alpha \gamma t\right]
$$

Proceeding as in Lemma 5.11 we have now instead of the inequality (5.41) the inequality,

$$
\begin{aligned}
\sum_{m+k=N}^{\infty} A_{m, k} \leqslant & \frac{\lambda_{\sigma}}{\alpha\left(\lambda_{\sigma}-\alpha \gamma\right)} \exp \left[\frac{4 D}{h^{2}}\{\cosh (\theta h)-1\} t-\alpha \gamma t\right] \\
& \times\left\{\frac{\lambda_{\sigma}\left[1-\delta_{h, \sigma, \alpha}(\theta)\right]}{\left(\lambda_{\sigma}-\alpha \gamma\right)(1-\alpha)}\right\}^{N} .
\end{aligned}
$$

The inequality (5.46) evidently also holds for $N=0$. In view of (5.43) the sum of the RHS of (5.46) w.r.t. $N$ is convergent, whence we obtain (5.44).

Next we wish to take the continuum limit $h \rightarrow 0$ of Lemma 5.12. In order to do this we find the continuum limit of the function $\delta_{h, \sigma}(\theta)$ defined by (5.40). 
Lemma 5.13. For the function $\delta_{h, \sigma}(\theta)$ of (5.40) there is the limit,

$$
\lim _{h \rightarrow 0} \delta_{h, \sigma}(\theta)=2 D \theta \sqrt{\lambda_{\sigma}} /\left[\sigma^{2}+2 \sqrt{2 \lambda_{\sigma} D}\right]\left[\sqrt{\lambda_{\sigma}}+\theta \sqrt{D / 2}\right] .
$$

Proof. Let $Z_{h}(t)$ be the walk defined by (5.30) with the diffusion constant $D$ replaced by $D \cosh (\theta h / 2)$. Let $g$ be a bounded function on the state space of the chain determined by (5.30) and define $v_{h}(z)$ by

$$
v_{h}(z)=\int_{0}^{\infty} E\left[g\left(Z_{h}(t)\right) ; t<\tau_{h} \mid Z_{h}(0)=z\right] e^{-\lambda t} \lambda d t,
$$

where $\lambda>0$ is an arbitrary parameter. It follows from (5.31), (5.32), (5.33) that $v_{h}(z)$ satisfies the difference equation,

$$
\frac{2 D \cosh (\theta h / 2)}{h^{2}}\left[2 v_{h}(z)-v_{h}(z+h)-v_{h}(z-h)\right]+\lambda v_{h}(z)=\lambda g(z), \quad z>0,
$$

$$
\frac{4 D \cosh (\theta h / 2)}{h^{2}}\left[v_{h}(0)-v_{h}(h)\right]+\left(\frac{\sigma^{2}}{h}+\lambda\right) v_{h}(0)=\lambda g(0) .
$$

For $z^{\prime} \in h \mathbf{Z}, z^{\prime}>0$, let $v_{h}(z)=G_{\lambda, h}\left(z, z^{\prime}\right)$ be the solution of (5.48) when $\lambda g(z)$ is the Kronecker delta $\lambda g(z)=h^{-1} \delta\left(z-z^{\prime}\right)$. By standard calculation one sees that $G_{\lambda, h}$ is given by the formula,

$$
\begin{aligned}
G_{\lambda, h}\left(z, z^{\prime}\right)= & B_{h}\left(z^{\prime}\right)\left[1+v h^{2}-h \sqrt{2 v+v^{2} h^{2}}\right]^{z / h}, z \geqslant z^{\prime}, \\
G_{\lambda, h}\left(z, z^{\prime}\right)= & C_{h}\left(z^{\prime}\right)\left[1+v h^{2}+h \sqrt{2 v+v^{2} h^{2}}\right]^{z / h} \\
& +E_{h}\left(z^{\prime}\right)\left[1+v h^{2}-h \sqrt{2 v+v^{2} h^{2}}\right]^{z / h}, \quad 0 \leqslant z \leqslant z^{\prime},
\end{aligned}
$$

where $v=\lambda / 4 D \cosh (\theta h / 2)$,

$$
C_{h}\left(z^{\prime}\right)\left[1-\frac{h}{2}\left\{\sqrt{2 v+v^{2} h^{2}}-v h\right\}\right]=\frac{v\left[1+v h^{2}-h \sqrt{2 v+v^{2} h^{2}}\right]^{z^{\prime} / h}}{\lambda\left[v h+\sqrt{2 v+v^{2} h^{2}}\right]}
$$




$$
\begin{gathered}
E_{h}\left(z^{\prime}\right)=-\left\{\frac{\sigma^{2} / 4 D \cosh (\theta h / 2)-\sqrt{2 v+v^{2} h^{2}}}{\sigma^{2} / 4 D \cosh (\theta h / 2)+\sqrt{2 v+v^{2} h^{2}}}\right\} C_{h}\left(z^{\prime}\right), \\
B_{h}\left(z^{\prime}\right)=C_{h}\left(z^{\prime}\right)\left[1+v h^{2}+h \sqrt{2 v+v^{2} h^{2}}\right]^{2 z^{\prime} / h}+E_{h}\left(z^{\prime}\right) .
\end{gathered}
$$

Note that in view of the fact that

$$
\frac{h}{2}\left\{\sqrt{2 v+v^{2} h^{2}}-v h\right\}<1,
$$

the coefficient of $C_{h}\left(z^{\prime}\right)$ in (5.49) is strictly positive. It is evident that the limit of the function $G_{\lambda, h}$ exists as $h \rightarrow 0$ and is given by the formula,

$$
\begin{gathered}
G_{\lambda}\left(z, z^{\prime}\right)=B\left(z^{\prime}\right) \exp \left[-z \sqrt{\frac{\lambda}{2 D}}\right], \quad z \geqslant z^{\prime}, \\
G_{\lambda}\left(z, z^{\prime}\right)=C\left(z^{\prime}\right) \exp \left[z \sqrt{\frac{\lambda}{2 D}}\right]+E\left(z^{\prime}\right) \exp \left[-z \sqrt{\frac{\lambda}{2 D}}\right], 0<z \leqslant z^{\prime},
\end{gathered}
$$

where

$$
\begin{aligned}
& C\left(z^{\prime}\right)=\frac{1}{2 \sqrt{2 \lambda D}} \exp \left[-z^{\prime} \sqrt{\frac{\lambda}{2 D}}\right], \\
& E\left(z^{\prime}\right)=-\frac{\sigma^{2}-2 \sqrt{2 \lambda D}}{\sigma^{2}+2 \sqrt{2 \lambda D}} C\left(z^{\prime}\right), \\
& B\left(z^{\prime}\right)=C\left(z^{\prime}\right) \exp \left[2 z^{\prime} \sqrt{\frac{\lambda}{2 D}}\right]+E\left(z^{\prime}\right) .
\end{aligned}
$$

Suppose now $g: \mathbf{R}^{+} \rightarrow \mathbf{R}$ is a continuous bounded function satisfying $g(0)=0$. Then the solution of $(5.48)$ is given by the formula,

$$
v_{h}(z)=\lambda \sum_{z^{\prime} \in h \mathbf{Z}, z^{\prime}>0} h G_{\lambda, h}\left(z, z^{\prime}\right) g\left(z^{\prime}\right) .
$$

Evidently one has that

$$
\lim _{h \rightarrow 0} v_{h}(z)=v(z)=\lambda \int_{0}^{\infty} G_{\lambda}\left(z, z^{\prime}\right) g\left(z^{\prime}\right) d z^{\prime}
$$


Note also that $v(z)$ is the solution to the boundary value problem which is the continuum limit of (5.48),

$$
\begin{gathered}
-2 D v^{\prime \prime}(z)+\lambda v(z)=\lambda g(z), \quad z>0, \\
v^{\prime}(0)=\sigma^{2} v(0) / 4 D .
\end{gathered}
$$

Now to compute the limit of $\delta_{h, \sigma}(\theta)$ as $h \rightarrow 0$ we observe that $\delta_{h, \sigma}(\theta)=$ $v_{h}(0)$ where the function $g$ is given by

$$
g(z)=1-\exp [-\theta z / 2], \quad z \geqslant 0 .
$$

Since $g(0)=0$ we have that

$$
\lim _{h \rightarrow 0} \delta_{h, \sigma}(\theta)=\lambda \int_{0}^{\infty} G_{\lambda}\left(0, z^{\prime}\right)\left[1-e^{-\theta z^{\prime} / 2}\right] d z^{\prime} .
$$

One can easily compute the RHS of this last equation from (5.52), (5.53), to obtain (5.47).

Remark 2. A boundary value problem similar to (5.54) has been previously studied in the context of particle systems (see ref. 17).

Lemma 5.13 enables us to take the continuum limit of Lemma 5.12. We do this under the assumption that solutions of the discretized equation (5.1) converge in distribution to solutions of the continuous equation,

$$
\frac{\partial u}{\partial t}(x, t)=D u_{x x}+\gamma u(1-u)+\sigma \sqrt{u(1-u)} W(x, t)
$$

as $h \rightarrow 0$.

Lemma 5.14. Let $u(x, t)$ be the solution of (5.55) with initial condition $u(x, 0)=1, x<0, u(x, 0)=0, x \geqslant 0$. Let $\alpha$ satisfy the inequalities, $0<\alpha<1, \alpha \gamma<\lambda_{\sigma}$. Define $\delta_{\sigma, \alpha}(\theta)$ by

$$
\delta_{\sigma, \alpha}(\theta)=2 D \theta \sqrt{\lambda} /\left[\sigma^{2}+2 \sqrt{2 \lambda D}\right][\sqrt{\lambda}+\theta \sqrt{D / 2}]
$$

where $\lambda=\lambda_{\sigma}-\alpha \gamma>0$. Suppose $\alpha$ also satisfies the inequality,

$$
\alpha\left[1+\gamma / \lambda_{\sigma}\right]<\delta_{\sigma, \alpha}(\theta) .
$$


Then there is the inequality,

$$
\langle 1-u(x, t)\rangle \leqslant \frac{\alpha^{-1} \lambda_{\sigma}(1-\alpha)}{\alpha^{2} \gamma+\lambda_{\sigma} \delta_{\sigma, \alpha}(\theta)-\alpha\left(\gamma+\lambda_{\sigma}\right)} \exp \left[\theta x+2 D \theta^{2} t-\alpha \gamma t\right] .
$$

Proof. The result follows from Lemma 5.12, Lemma 5.13, the identity (5.2) and Lemma 5.7.

The following lemma now completes the proof of inequality (1.7) of Theorem 1.2.

Lemma 5.15. Let $c(\sigma)$ be the wave speed for the equation (5.55) as defined in Lemma 5.3. Then $c(\sigma)>0$ for $\sigma>0$ and there is the inequality,

$$
\liminf _{\sigma \rightarrow \infty} \sigma^{2} c(\sigma) \geqslant 2 D \gamma
$$

Proof. We apply the argument of Lemma 5.4 and we use Lemma 5.14. Let $\alpha(\sigma, \theta)$ be the value of $\alpha$ determined by equality in (5.56). Then one has that

$$
\lim _{\theta \rightarrow 0} \alpha(\sigma, \theta) / \theta=2 D /\left[\sigma^{2}+2 \sqrt{2 \lambda D}\right]\left[1+\gamma / \lambda_{\sigma}\right]=\alpha(\sigma)
$$

By choosing $\theta$ in (5.57) to be sufficiently small and independent of $t$ we see that $c(\sigma) \geqslant \alpha(\sigma) \gamma$ provided $0<\alpha(\sigma)<1, \alpha(\sigma) \gamma<\lambda_{\sigma}$. By choosing $\lambda_{\sigma}=$ $\sigma^{2}$ and letting $\sigma \rightarrow \infty$ we obtain the inequality (5.58).

\section{ACKNOWLEDGMENTS}

We are grateful to C. Mueller, L. Sander, P. Smereka and R. Ziff for enlightening discussions, and to three anonymous referees for helpful remarks. This research was supported in part by NSF awards DMS0138519 (Conlon) and DMS-0244419 (Doering, Conlon et al.).

\section{REFERENCES}

1. D. Aronson and H. Weinberger, Nonlinear diffusion in population genetics, combustion, and nerve propagation, in Partial Differential Equations and Related Topics, J. Goldstein, ed. (Springer, New York, 1975), Lecture Notes in Mathematics no. 446, pp. 5-49.

2. M. Bramson, Convergence of solutions of the Kolmogorov equation to travelling waves, Mem. Amer. Math. Soc. 44:285 (1983). 
3. E. Brunet and B. Derrida, Shift in the velocity of a front due to a cutoff, Phys. Rev. E. 56:2597-2604 (1997).

4. C. R. Doering, A stochastic partial differential equation with multiplicative noise, Phys. Lett. A 122:133-139 (1987).

5. C. R. Doering, C. Mueller and P. Smereka, Interacting particles, the stochastic FisherKolmogorov-Petrovsky-Piscunov equation and duality, Physica A 325:243-259 (2003).

6. U. Ebert and W. van Saarloos, Front propagation into unstable states: Universal algebraic convergence towards uniformly translating pulled fronts, Physica D 146:1-99 (2000).

7. P. Fife and J.B. McLeod, The approach of solutions of nonlinear diffusion equations to travelling wave front solutions, Arch. Rat. Mech. Anal. 65:335-362 (1977).

8. R. A. Fisher, The wave of advance of advantageous genes, Ann. Eugen. 7:355-369 (1937).

9. D. A. Kessler, Z. Ner, and L. M. Sander, Front propagation: Precursors, cutoffs, and structural stability, Phys. Rev. E 58:107-114 (1998).

10. A. Kolmogorov, I. Petrovsky, and N. Piscunov, Étude de l'équation de la diffusion avec croissance de la quantité de matière et son application à un problème biologique, Moscou Universitet Bull. Math. 1:1-25 (1937).

11. T. Liggett, Interacting Particles Systems (Springer-Verlag, Berlin, 1985).

12. H. McKean, Application of Brownian motion to the equation of Kolmogorov-PetrowskiiPiskunov, Comm. Pure Appl. Math. 28:323-331 (1975).

13. C. Mueller and R. Sowers, Random Travelling Waves for the KPP Equation with Noise, J. Functional Analysis 128:439-498 (1995).

14. C. Mueller and R. Tribe, Stochastic p.d.e.'s arising from the long range contact and long range voter processes, Probab. Theory Relat. Fields 102:519-545 (1995).

15. L. Pechenik and H. Levine, Interfacial velocity corrections due to multiplicative noise, Phys. Rev E 59:3893-3900 (1999).

16. E. Perkins, Super-Brownian motion and critical spatial stochastic systems, Canad. Math. Bull. 47:280-297 (2004).

17. V. Privman, C. Doering and H. Frisch, Crossover from rate-equation to diffusioncontrolled kinetics in two-particle coagulation, Phys. Rev. E. 48:846-851 (1993).

18. T. Shiga, Two contrasting properties of solutions for one-dimensional stochastic partial differential equations, Can. J. Math. 46:415-437 (1994).

19. T. Shiga and K. Uchiyama, Stationary states and the stability of the stepping stone model involving mutation and selection, Probab. Theory Relat. Fields 73:87-117 (1986).

20. R. Tribe, Large time behavior of interface solutions to the heat equation with FisherWright white noise, Probab. Theory Relat. Fields 102:289-311 (1995).

21. J. Xin, Front propagation in heterogeneous media, SIAM Review 42:161-230 (2000). 